THE ASTROPHYSICAL JouRNAL, 567:515-531, 2002 March 1

(c) 2002. The American Astronomical Society. All rights reserved. Printed in U.S.A.

\title{
DETERMINATION OF NUCLEOSYNTHETIC YIELDS OF SUPERNOVAE AND VERY MASSIVE STARS FROM ABUNDANCES IN METAL-POOR STARS
}

\author{
Y.-Z. QIAN ${ }^{1}$ AND G. J. WASSERBURG ${ }^{2}$ \\ Received 2001 August 20; accepted 2001 October 24
}

\begin{abstract}
We determine the yields of the elements from $\mathrm{Na}$ to Ni for Type II supernovae (SNe II) and the yield patterns of the same elements for Type Ia supernovae (SNe Ia) and very massive $\left(\gtrsim 100 M_{\odot}\right)$ stars (VMSs) using a phenomenological model of stellar nucleosynthesis and the data on a number of stars with $-4 \lesssim[\mathrm{Fe} / \mathrm{H}] \lesssim-3$, a single star with $[\mathrm{Fe} / \mathrm{H}]=-2.04$, and the Sun. We consider that there are two distinct kinds of SNe II: the high-frequency SNe $\mathrm{II}(H)$ and the low-frequency $\mathrm{SNe} \operatorname{II}(L)$. We also consider that VMSs were the dominant first-generation stars formed from big bang debris. The yield patterns of $\mathrm{Na}$ to $\mathrm{Ni}$ for $\mathrm{SNe} \mathrm{II}(H), \mathrm{II}(L)$, and Ia and VMSs appear to be well defined. It is found that $\mathrm{SNe} \mathrm{II}(H)$ produce almost none of these elements; that $\mathrm{SNe} \mathrm{II}(L)$ can account for the entire solar inventory of $\mathrm{Na}, \mathrm{Mg}, \mathrm{Si}, \mathrm{Ca}, \mathrm{Ti}$, and $\mathrm{V}$; and that compared with $\mathrm{SNe} \mathrm{II}(L)$, VMSs underproduce $\mathrm{Na}, \mathrm{Al}, \mathrm{V}$, $\mathrm{Cr}$, and $\mathrm{Mn}$, overproduce $\mathrm{Co}$, but otherwise have an almost identical yield pattern. A comparison is made between the yield patterns determined here from the observational data and those calculated from ab initio models of nucleosynthesis in SNe II and VMSs. We show that the evolution of the "heavy" elements in the universe relative to Fe involves three distinct stages. The earliest stage is in the domain of $[\mathrm{Fe} / \mathrm{H}]<-3$ and is governed by VMS activities with some small contributions from $\mathrm{SNe}$ II, all of which are dispersed in a dilution mass of $M_{\mathrm{dil}}^{\mathrm{VMS}} \sim 10^{6}-10^{7} M_{\odot}$. The beginning of the second stage is marked by the cessation of VMS activities and the onset of major formation of normal stars (with masses of $\sim 1-60 M_{\odot}$ ) at $[\mathrm{Fe} / \mathrm{H}] \approx-3$. The cessation of VMS activities causes the dilution mass for SN II contributions to drop sharply to $M_{\mathrm{dil}}^{\mathrm{SNII}} \approx 3 \times 10^{4} M_{\odot}$. The subsequent quasi-continuous chemical evolution until $[\mathrm{Fe} / \mathrm{H}] \sim-1$ is governed by $\mathrm{SNe} \mathrm{II}(H)$, which produce mainly the heavy $r$-process elements above $\mathrm{Ba}$, and $\mathrm{SNe} \mathrm{II}(L)$, which produce essentially all the other elements. The third stage starts with the onset of SN Ia contributions to mainly the $\mathrm{Fe}$ group elements at $[\mathrm{Fe} / \mathrm{H}] \sim-1$. The domain of $[\mathrm{Fe} / \mathrm{H}]>-1$ is then governed by contributions from $\mathrm{SNe} \mathrm{II}(H), \mathrm{II}(L)$, and Ia and low-mass stars. It is shown that the abundances of non-neutron capture elements in stars with $[\mathrm{Fe} / \mathrm{H}] \leq 0$ and those of $r$ process elements in stars with $[\mathrm{Fe} / \mathrm{H}]<-1$ can be well represented by the sum of the distinct components in the phenomenological model. The proposed evolutionary sequence is directly related to the problems of early aggregation and dispersion of baryonic matter and to the onset of formation and chemical evolution of galaxies. It is argued that the prompt inventory governed by VMS contributions should represent the typical composition of dispersed baryonic matter in the universe, and that normal galactic evolution should begin in matter with $[\mathrm{Fe} / \mathrm{H}] \approx 3$.

Subject headings: Galaxy: abundances - Galaxy: evolution -

nuclear reactions, nucleosynthesis, abundances - stars: abundances stars: Population II
\end{abstract}

\section{INTRODUCTION}

In this paper we discuss the nucleosynthetic yields of supernovae ( $\mathrm{SNe}$ ) and very massive stars (VMSs, with masses of $\gtrsim 100 M_{\odot}$ ) based on the observed abundances in stars with $-4 \lesssim[\mathrm{Fe} / \mathrm{H}]<-1$. It was considered in our previous works (Wasserburg \& Qian 2000a, hereafter WQ00a; Wasserburg \& Qian 2000b; Qian \& Wasserburg 2001a, 2001c, hereafter QW01c) that VMSs were formed from big bang debris and provided an initial or prompt $(P)$ inventory of $\mathrm{Fe}$ and associated elements up to $[\mathrm{Fe} / \mathrm{H}] \approx-3$. It was assumed that normal stars (with masses of $\sim 1-60 M_{\odot}$ ) could only be formed at $[\mathrm{Fe} / \mathrm{H}] \gtrsim$ -3 . Subsequent evolution of normal stars led to Type II $\mathrm{SNe}$ (SNe II) and at much later times (corresponding to $[\mathrm{Fe} / \mathrm{H}] \gtrsim-1$ ) also to Type Ia $\mathrm{SNe}$ (SNe Ia), all of which provided further chemical enrichment of the interstellar

\footnotetext{
${ }^{1}$ School of Physics and Astronomy, University of Minnesota, Minneapolis, MN 55455; qian@physics.umn.edu.

${ }^{2}$ The Lunatic Asylum, Division of Geological and Planetary Sciences, MS 170-25, California Institute of Technology, Pasadena, CA 91125.
}

medium (ISM) beyond the $P$ inventory. The special status of $[\mathrm{Fe} / \mathrm{H}] \approx-3$ in chemical evolution of the ISM was identified based on a sharp increase in the abundances of heavy $r$-process elements ( $\mathrm{Ba}$ and above) at this metallicity (WQ00a). This is shown in Figure 1 for Ba. The sharp increase was attributed to the rapid occurrence of highfrequency $\mathrm{SNe} \mathrm{II}(H)$ that produce mainly the heavy $r$ process elements but no Fe. The Fe enrichment of the ISM at $-3<[\mathrm{Fe} / \mathrm{H}]<-1$ was attributed to the low-frequency $\mathrm{SNe} \mathrm{II}(L)$ and that at $[\mathrm{Fe} / \mathrm{H}] \gtrsim-1$ to both $\mathrm{SNe} \mathrm{II}(L)$ and Ia. In addition to contributing $\approx \frac{1}{3}$ of the solar Fe inventory, $\mathrm{SNe} \mathrm{II}(L)$ are mainly responsible for the light $r$-process elements (Ba and below). QW01c have derived the $P$ inventory and $\mathrm{SN} \mathrm{II}(H)$ and $\mathrm{II}(L)$ yields of $r$-process elements from the observed abundances in two stars with $[\mathrm{Fe} / \mathrm{H}] \approx-3$ and the solar $r$-process abundances. They have shown that the abundances calculated from the threecomponent model including the $P$ inventory and the contributions from $\mathrm{SNe} \mathrm{II}(H)$ and $\mathrm{II}(L)$ were in good agreement with several independent data sets for a large number of $r$-process elements over the wide range of 


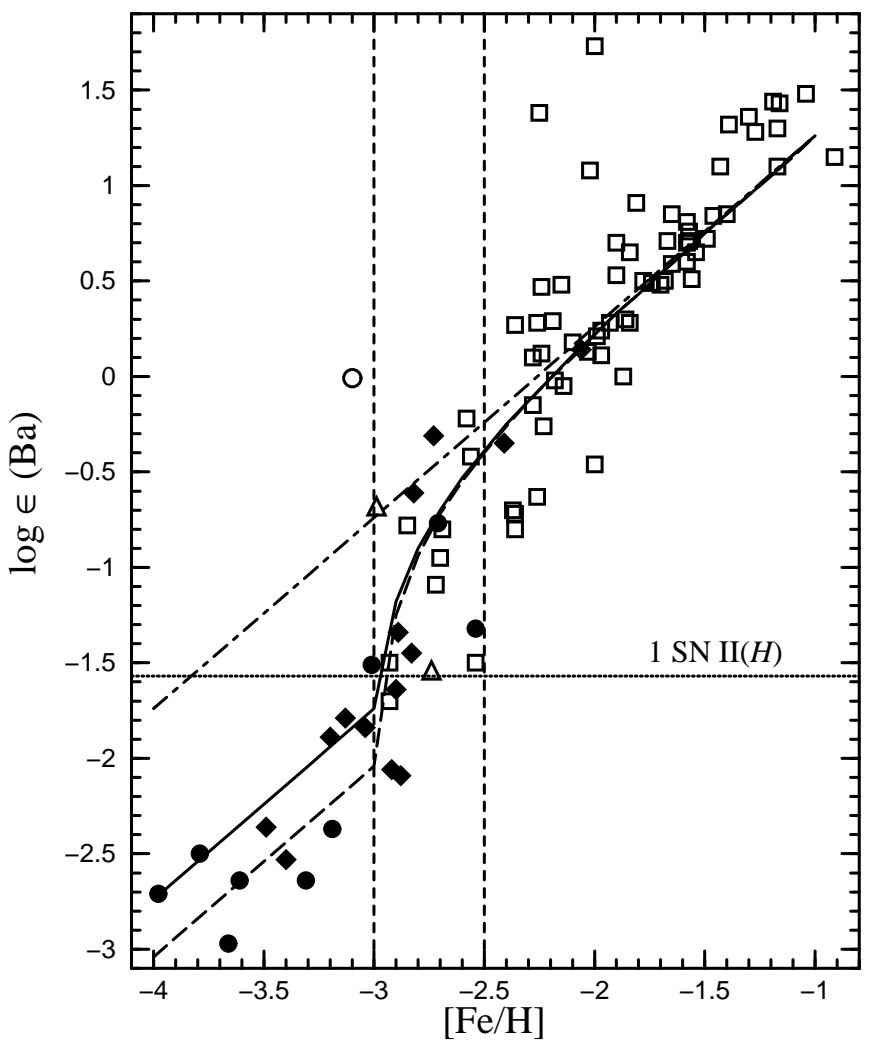

FIG. 1.-Data ( filled circles: Ryan, Norris, \& Beers 1996; Norris et al. 2001; filled diamonds: McWilliam et al. 1995; McWilliam 1998; open squares: Burris et al. 2000; lower and upper open triangles: HD 122563 and HD 115444, Westin et al. 2000; open circle: CS 22892-052, Sneden et al. $2000)$ on $\log \epsilon(\mathrm{Ba})$ as a function of $[\mathrm{Fe} / \mathrm{H}]$. The Ba yield of a single $\mathrm{SN} \mathrm{II}(H)$ is shown as the dotted line and that of a single $\mathrm{SN} \mathrm{II}(L)$ is 1.1 dex above this. The straight dot-dashed line represents the evolutionary trend for production only by $\mathrm{SNe} \mathrm{II}(H)$ and $\mathrm{II}(L)$ with a frequency ratio of 10:1. The extension of this line below $[\mathrm{Fe} / \mathrm{H}] \sim-2.5$ corresponds to greater than standard dilution masses but no other $\mathrm{Ba}$ or $\mathrm{Fe}$ sources. The solid and long-dashed curves are for the continuous model of evolution with VMS activities and the associated large dilution mass at $[\mathrm{Fe} / \mathrm{H}]<-3$. It is considered that VMSs produce no $\mathrm{Ba}$ but dominate the $\mathrm{Fe}$ production until the cessation of VMS activities at $[\mathrm{Fe} / \mathrm{H}] \approx-3$. The solid and longdashed curves assume that $\mathrm{SNe} \operatorname{II}(L)$ contributed $10 \%$ and $5 \%$, respectively, of the $\mathrm{Fe}$ at $[\mathrm{Fe} / \mathrm{H}]<-3$. The continuous approximation fails and a discrete model must be used in the transition region between the two vertical short-dashed lines (see discussion of Fig. 5).

$-3 \lesssim[\mathrm{Fe} / \mathrm{H}]<-1$. The same agreement was also achieved for $\mathrm{Sr}, \mathrm{Y}, \mathrm{Zr}$, and $\mathrm{Ba}$ when the standard solar $r$-process abundances of these elements (Arlandini et al. 1999 ) were increased by factors of $\approx 2-6$. In general, the model of QW01c gave a satisfactory description of the chemical evolution of all the $r$-process elements over $-3 \lesssim[\mathrm{Fe} / \mathrm{H}]<-1$, which corresponds to a period of $\approx 3 \times 10^{9} \mathrm{yr}$ in the early Galactic history.

Here we extend the model of QW01c to the elements from $\mathrm{Na}$ to $\mathrm{Ni}$ and derive the relevant yields of $\mathrm{SNe} \mathrm{II}(H)$ and $\mathrm{II}(L)$ and the yield pattern of SNe Ia $(\S 2)$. We also infer the yield pattern of VMSs from the observed abundances in stars with $-4 \lesssim[\mathrm{Fe} / \mathrm{H}] \lesssim-3(\S 3)$. It will be shown that $\mathrm{SNe} \mathrm{II}(H)$ produce almost none of the elements from $\mathrm{Na}$ to $\mathrm{Ni}$; that $\mathrm{SNe} \mathrm{II}(L)$ can account for the entire solar inventory of $\mathrm{Na}, \mathrm{Mg}, \mathrm{Si}, \mathrm{Ca}, \mathrm{Ti}$, and $\mathrm{V}$; and that compared with $\mathrm{SNe}$ $\mathrm{II}(L)$, VMSs underproduce $\mathrm{Na}, \mathrm{Al}, \mathrm{V}, \mathrm{Cr}$, and $\mathrm{Mn}$, overproduce $\mathrm{Co}$, but otherwise have an almost identical yield pattern. We reinterpret the metallicity of $[\mathrm{Fe} / \mathrm{H}] \approx-3$ as corresponding to the cessation of VMS activities and the onset of major formation of normal stars. It will be shown that VMSs dominated the production of $\mathrm{Na}$ to $\mathrm{Ni}$ at $[\mathrm{Fe} / \mathrm{H}] \lesssim-3$ and that explosions of these objects could induce mixing within $\sim 10^{6}-10^{7} M_{\odot}$ of the ISM, to be compared with $\approx 3 \times 10^{4} M_{\odot}$ for SNe II. A model for general chemical evolution of the ISM starting from a zerometallicity state will be presented (§4). The wide range in yields calculated from nucleosynthetic models for SNe II and VMSs will be discussed in light of the rather constant and metallicity-independent yield patterns as determined here from the observed abundances in metal-poor stars ( $\$ 5$ ). The cosmological implications of the results presented here will also be discussed $(\S 5)$.

\section{THREE-COMPONENT MODEL AND THE $P$ INVENTORY AND SN YIELDS}

We consider a homogeneous mass of gas and assume that stars formed from this gas have the composition of the gas. The chemical evolution of an element $\mathrm{E}$ in this gas is specified by the initial number of $\mathrm{E}$ atoms per $\mathrm{H}$ atom in the gas and the number of $\mathrm{E}$ atoms per $\mathrm{H}$ atom added from various $\mathrm{SNe}$ II to the gas. As shown by QW01c, if each distinct kind of SN II has a fixed relative yield pattern, then the inventory of $\mathrm{E}$ atoms may be simply described by the effective numbers of SNe II that contributed to the gas. The effective numbers are calculated by assuming constant yields and a standard dilution mass for SNe II. The results of astration, fragmentation, and merging of different gas masses, as well as the effects of variable SN II yields (but with fixed yield patterns), are subsumed in the representation of the effective numbers of contributing SNe II. By using this approach, it was shown that the abundances of $r$-process and related elements ( $\mathrm{Sr}$ and above) in stars with $-3 \lesssim[\mathrm{Fe} / \mathrm{H}]<-1$ can be described by a three-component model including the $P$ inventory and the contributions from $\mathrm{SNe} \operatorname{II}(H)$ and $\mathrm{II}(L)$ (QW01c). For a star formed from an ISM with a number $n_{H}$ of contributing $\mathrm{SNe} \mathrm{II}(H)$ and a number $n_{L}$ of contributing $\mathrm{SNe} \mathrm{II}(L)$, the abundance of an element $\mathrm{E}$ in the star is given by

$$
10^{\log \epsilon(\mathrm{E})}=10^{\log \epsilon(\mathrm{E})}+n_{H} \times 10^{\log \epsilon_{H}(\mathrm{E})}+n_{L} \times 10^{\log \epsilon_{L}(\mathrm{E})},
$$

where $\log \epsilon_{P}(\mathrm{E}), \log \epsilon_{H}(\mathrm{E})$, and $\log \epsilon_{L}(\mathrm{E})$ represent the $P$ inventory and the $\mathrm{SN} \mathrm{II}(H)$ and $\mathrm{II}(L)$ yields of $\mathrm{E}$, respectively. Here and below, the standard spectroscopic notation $\log \epsilon(\mathrm{E}) \equiv \log (\mathrm{E} / \mathrm{H})+12$ is used. The parameter $\log \epsilon_{H}(\mathrm{E})$ or $\log \epsilon_{L}(\mathrm{E})$ corresponds to the abundance of $\mathrm{E}$ in the ISM resulting from a single $\mathrm{SN} \mathrm{II}(H)$ or $\mathrm{II}(L)$ for a standard dilution mass $\left(M_{\mathrm{dil}}^{\mathrm{SNII}} \approx 3 \times 10^{4} M_{\odot}\right)$ of baryonic matter that is free of "metals" and dominantly composed of $\mathrm{H}$. This dilution mass is fixed by the frequency of $\mathrm{SNe} \mathrm{II}(H)$ as required for replenishment of fresh radioactive ${ }^{182} \mathrm{Hf}$ in the ISM (e.g., Wasserburg, Busso, \& Galllino 1996; QW01c) and is consistent with the typical total amount of ISM swept up by an SN II remnant (e.g., Thornton et al. 1998).

As the heavy $r$-process elements above $\mathrm{Ba}$, e.g., $\mathrm{Eu}$, are exclusively produced by $\mathrm{SNe} \mathrm{II}(H)$, the number $n_{H}$ for the star can be obtained from its observed $\log \epsilon(\mathrm{Eu})$ :

$$
n_{H}=10^{\log \epsilon(\mathrm{Eu})-\log \epsilon_{H}(\mathrm{Eu})}=10^{\log \epsilon(\mathrm{Eu})+2.48},
$$

where $\log \epsilon_{H}(\mathrm{Eu})=-2.48(\mathrm{QW01c})$ is used. Likewise, as additions of $\mathrm{Fe}$ at $-3<[\mathrm{Fe} / \mathrm{H}]<-1$ beyond the $P$ inven- 
tory are made only by $\mathrm{SNe} \mathrm{II}(L)$, the number $n_{L}$ for the star can be obtained from its observed $\log \epsilon(\mathrm{Fe})$ :

$$
n_{L}=\frac{10^{\log \epsilon(\mathrm{Fe})}-10^{\log \epsilon P(\mathrm{Fe})}}{10^{\log \epsilon_{L}(\mathrm{Fe})}},
$$

where $\log \epsilon_{P}(\mathrm{Fe})=4.51$ and $\log \epsilon_{L}(\mathrm{Fe})=5.03$ (QW01c). By using $[\mathrm{Fe} / \mathrm{H}] \equiv \log \epsilon(\mathrm{Fe})-\log \epsilon_{\odot}(\mathrm{Fe})$ with $\log \epsilon_{\odot}(\mathrm{Fe})=$ 7.51 (Anders \& Grevesse 1989), equation (3) can be rewritten as

$$
n_{L}=\frac{10^{[\mathrm{Fe} / \mathrm{H}]}-10^{[\mathrm{Fe} / \mathrm{H}] P}}{10^{[\mathrm{Fe} / \mathrm{H}]_{L}}}=10^{[\mathrm{Fe} / \mathrm{H}]+2.48}-10^{-0.52},
$$

where $[\mathrm{Fe} / \mathrm{H}]_{P}=-3$ and $[\mathrm{Fe} / \mathrm{H}]_{L}=-2.48$ are used. The parameters $\log \epsilon_{P}, \log \epsilon_{H}$, and $\log \epsilon_{L}$ for the pertinent elements were obtained from the data on two stars with $[\mathrm{Fe} / \mathrm{H}] \approx-3$ and the solar $r$-process abundances (QW01c). It was shown that the abundances calculated from the above model for a large number of $r$-process elements were in good agreement with several independent data sets over the wide range of $-3 \lesssim[\mathrm{Fe} / \mathrm{H}]<-1$. The same agreement was also achieved for $\mathrm{Sr}, \mathrm{Y}, \mathrm{Zr}$, and $\mathrm{Ba}$ when the standard solar $r$-process abundances of these elements (Arlandini et al. 1999) were increased by factors of $\approx 2-6$.

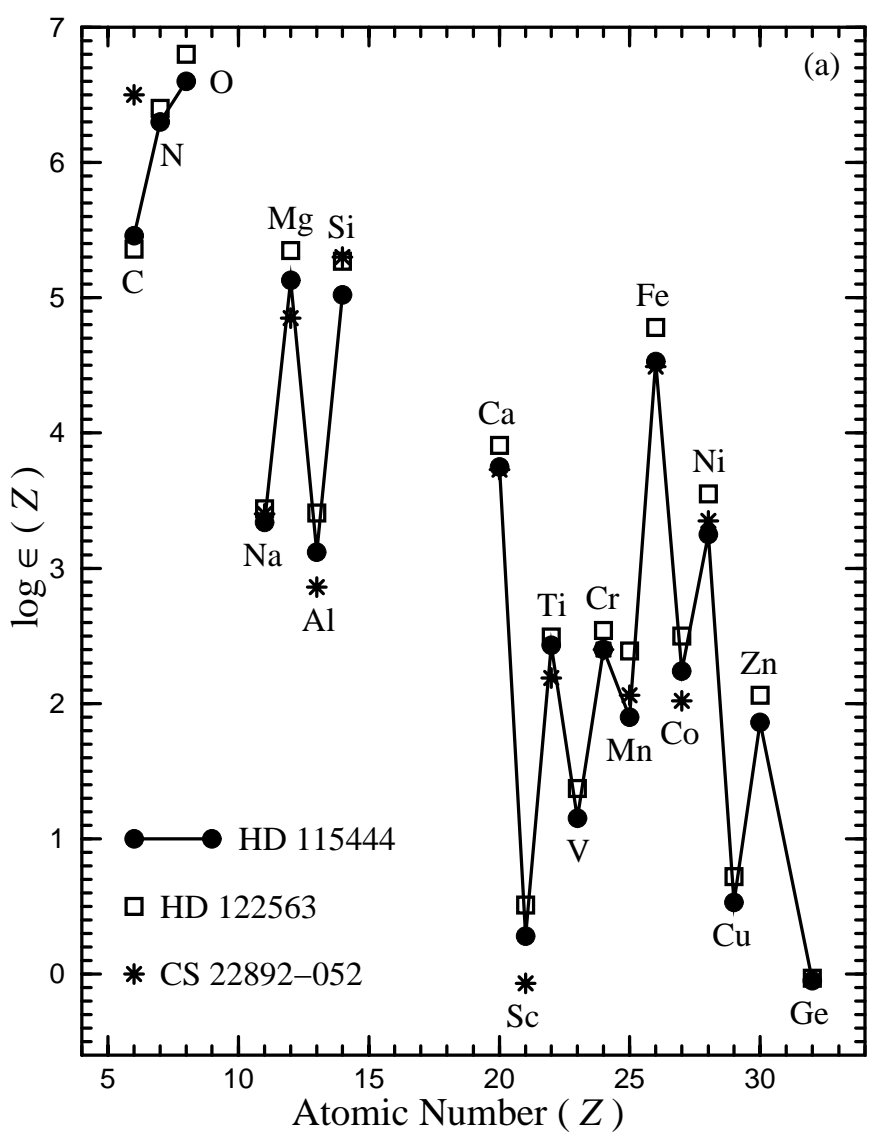

\subsection{Estimates of the P Inventory and SN II $(H)$ Yields}

We now extend the above three-component model to the elements below Sr. By the assumptions of the model, a fixed amount of ISM dilutes the ejecta from an SN II at $[\mathrm{Fe} / \mathrm{H}] \gtrsim-3$. It can be seen from equation (4) that for the standard dilution mass, stars with $[\mathrm{Fe} / \mathrm{H}] \approx-3$ have $n_{L} \approx$ 0 . Thus, the abundances in these stars only represent mixtures of the $P$ inventory and $\mathrm{SN} \mathrm{II}(H)$ contributions:

$$
10^{\log \epsilon(\mathrm{E})}=10^{\log \epsilon P(\mathrm{E})}+n_{H} \times 10^{\log \epsilon_{H}(\mathrm{E})} .
$$

Thus, with the value of $n_{H}$ obtained from equation (2), the parameters $\log \epsilon_{P}(\mathrm{E})$ and $\log \epsilon_{H}(\mathrm{E})$ can be determined from the data on any two stars with $[\mathrm{Fe} / \mathrm{H}] \approx-3$ but different $n_{H}$ values.

Figure $2 a$ shows the observed $\log \epsilon$ values of $\mathrm{C}$ to $\mathrm{Ge}$ (Westin et al. 2000; McWilliam et al. 1995) for HD 122563 $([\mathrm{Fe} / \mathrm{H}]=-2.74 ;$ open squares $), \mathrm{HD} 115444([\mathrm{Fe} / \mathrm{H}]=$ -2.99 ; filled circles), and CS 22892-052 $([\mathrm{Fe} / \mathrm{H}]=-3.03$; asterisks) with $n_{H} \approx 1,7$, and 36 , respectively. The typical uncertainties in the data shown in Figure $2 a$ are $\approx 0.1$ dex. It can be seen that the abundances of the elements above $\mathrm{C}$ are essentially the same for all three stars. In fact, when a uniform shift of -0.25 dex is applied to the data on HD 122563 so that all three sets of data correspond to essen-

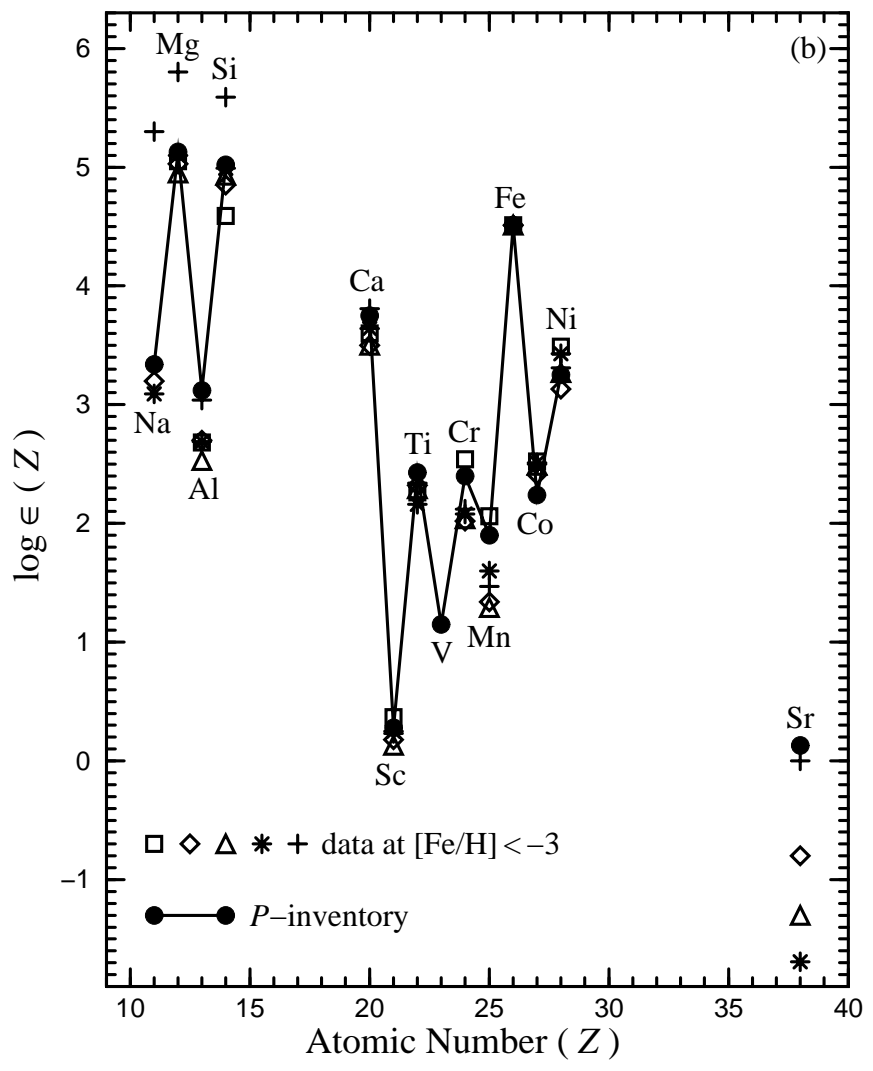

FIG. 2. (a) Data on three stars (open squares: HD 122563 with $[\mathrm{Fe} / \mathrm{H}]=-2.74$, Westin et al. 2000; filled circles connected with solid curves: HD 115444 with $[\mathrm{Fe} / \mathrm{H}]=-2.99$, Westin et al. 2000; asterisks: CS 22892-052 with $[\mathrm{Fe} / \mathrm{H}]=-3.03$, McWilliam et al. 1995) that are used to infer the $P$ inventory and SN II $(H)$ yields. Note the remarkable similarity in the abundances of all the elements above $\mathrm{C}$ for these stars with widely varying Eu abundances corresponding to SN II $(H)$ contributions for $n_{H} \approx 1,7$, and 36. (b) $P$ inventory ( filled circles connected with solid curves) calculated from the data in (a). The data on five stars (squares: $\mathrm{CD}-24^{\circ} 17504$ with $[\mathrm{Fe} / \mathrm{H}]=-3.37$ and no $\mathrm{Sr}$ data; diamonds: $\mathrm{CD}-38^{\circ} 245$ with $[\mathrm{Fe} / \mathrm{H}]=-3.98$; triangles: $\mathrm{CS} 22172-002$; asterisks: CS 22885-096; plus signs: CS 22949-037; McWilliam et al. 1995 for Na and Norris et al. 2001 for all the other elements) are shown for comparison. These data have been shifted from the observed abundances to pass through the same Fe abundance of $\log \epsilon(\mathrm{Fe})=\log \epsilon_{P}(\mathrm{Fe})=4.51$ and are almost indistinguishable from the $P$ inventory. There appear to be some anomalies at $\mathrm{Na}, \mathrm{Mg}$, and $\mathrm{Si}$ for one star (plus signs: CS 22949-037), some spread in Mn, and a wide scatter in Sr. 
tially the same $[\mathrm{Fe} / \mathrm{H}]$, significant differences are only found for $\mathrm{C}$ between the three stars and for Ge between HD 122563 and HD 115444. The differences for all the other elements are within the observational uncertainties. As these three stars have very different $n_{H}$ values, we conclude that $\mathrm{SNe} \mathrm{II}(H)$ produce very little of the elements from $\mathrm{N}$ to $\mathrm{Zn}$. The abundances of these elements in the three stars are then completely dominated by the $P$ inventory. We take the observed $\log \epsilon$ values of $\mathrm{N}$ to $\mathrm{Zn}$ for HD 115444 to be the corresponding $\log \epsilon_{P}$ values, thus defining the abundances in the $P$ inventory.

An upper limit on $\log \epsilon_{H}$ for $\mathrm{N}$ to $\mathrm{Zn}$ can be estimated from

$$
10^{\log \epsilon P(\mathrm{E})+\sigma(\mathrm{E})} \gtrsim 10^{\log \epsilon_{P}(\mathrm{E})}+n_{H}^{\mathrm{HD} 115444} \times 10^{\log \epsilon_{H}(\mathrm{E})},
$$

where $\sigma(\mathrm{E}) \approx 0.1 \mathrm{dex}$ is the uncertainty in the observed $\log \epsilon(\mathrm{E})$ value for HD 115444 and $n_{H}^{\mathrm{HD} 115444} \approx 7$. Equation (6) gives

$$
\log \epsilon_{H}(\mathrm{E}) \lesssim \log \epsilon_{P}(\mathrm{E})-1.43
$$

for $\mathrm{N}$ to $\mathrm{Zn}$. Thus, the contributions from an $\mathrm{SN} \mathrm{II}(H)$ with the standard dilution mass are small compared with the existing $P$ inventory for all the elements between $\mathrm{C}$ and $\mathrm{Ge}$.
The $\log \epsilon_{P}$ values and the upper limits on $\log \epsilon_{H}$ estimated in equation (7) are given in Table 1. It was argued that the extremely high value of $n_{H} \approx 36$ corresponding to the extremely high $r$-process abundances observed in CS 22892-052 is due to contamination of the surface of this star by the ejecta from the SN II $(H)$ explosion of a previous massive binary companion (Qian \& Wasserburg 2001b). The agreement between the observed $\log \epsilon$ values of all the elements from $\mathrm{Na}$ to $\mathrm{Ni}$ for CS 22892-052 and the corresponding $\log \epsilon_{P}$ values in Table 1 further supports the argument that the $\mathrm{SN} \mathrm{II}(H)$ yields of these elements are very low. On the other hand, the $\log \epsilon(\mathrm{C})$ value of CS 22892-052 is higher than those of HD 115444 and HD 122563 by $\approx 1$ dex (see Fig. 2a). This suggests that a significant amount of $\mathrm{C}$ may be produced in $\mathrm{SNe} \mathrm{II}(H)$ or that $\mathrm{C}$ was produced in CS 22892-052 during its evolution. The elements C, N, O, $\mathrm{Cu}, \mathrm{Zn}$, and $\mathrm{Ge}$ will not be discussed further (the evolution of $\mathrm{O}$ relative to $\mathrm{Fe}$ has been discussed in detail in Qian \& Wasserburg 2001a). Below we will focus on the elements from $\mathrm{Na}$ to $\mathrm{Ni}$ as there are high-quality data on these elements.

Figure $2 b$ compares the $P$ inventory (see Table 1 ) as determined above for $[\mathrm{Fe} / \mathrm{H}]=-3$ with the observational

TABLE 1

\begin{tabular}{|c|c|c|c|c|c|c|c|c|c|}
\hline $\begin{array}{l}Z^{\mathrm{a}} \\
(1)\end{array}$ & $\begin{array}{l}E \\
(2)\end{array}$ & $\begin{array}{c}\log \epsilon_{P}(\mathrm{E}) \\
\text { (3) }\end{array}$ & $\begin{array}{c}\log \epsilon_{H}(E) \\
\text { (4) }\end{array}$ & $\begin{array}{c}\log \epsilon_{L}(\mathrm{E}) \\
(5)\end{array}$ & $\begin{array}{c}\log \epsilon_{\odot}(\mathrm{E}) \\
\text { (6) }\end{array}$ & $\begin{array}{c}\alpha_{\odot, \mathrm{II}}(\mathrm{E}) \\
(7)\end{array}$ & $\begin{array}{c}\log \epsilon_{L}^{\text {corr }}(E) \\
(8)\end{array}$ & $\begin{array}{c}\alpha_{\odot}^{\text {corr III }}(E) \\
(9)\end{array}$ & $\begin{array}{c}\log \epsilon_{\odot, \mathrm{Ia}}(\mathrm{E}) \\
(10)\end{array}$ \\
\hline \multicolumn{10}{|c|}{ Group 1} \\
\hline $6 \ldots \ldots$ & $\mathrm{C}$ & $<5.46$ & $?$ & $?$ & 8.56 & $?$ & $?$ & $?$ & $-\infty$ \\
\hline $7 \ldots \ldots$ & $\mathrm{N}$ & 6.30 & $\lesssim 4.87$ & $?$ & 8.05 & $?$ & $?$ & $?$ & $-\infty$ \\
\hline $8 \ldots \ldots$ & $\mathrm{O}$ & 6.60 & $\lesssim 5.17$ & 6.93 & 8.93 & 1.00 & 6.93 & 1.00 & $-\infty$ \\
\hline \multicolumn{10}{|c|}{ Group 2} \\
\hline $11 \ldots \ldots$ & $\mathrm{Na}$ & 3.34 & $\lesssim 1.91$ & 4.24 & 6.33 & 0.81 & 4.33 & 1.00 & $-\infty$ \\
\hline $12 \ldots \ldots$ & $\mathrm{Mg}$ & 5.13 & $\lesssim 3.70$ & 5.62 & 7.58 & 1.09 & 5.58 & 1.00 & $-\infty$ \\
\hline $13 \ldots \ldots$ & $\mathrm{Al}$ & 3.12 & $\lesssim 1.69$ & 3.90 & 6.47 & 0.27 & 3.90 & 0.27 & $-\infty$ \\
\hline $14 \ldots \ldots$ & $\mathrm{Si}$ & 5.02 & $\lesssim 3.59$ & 5.47 & 7.55 & 0.84 & 5.55 & 1.00 & $-\infty$ \\
\hline $20 \ldots \ldots$ & $\mathrm{Ca}$ & 3.75 & $\lesssim 2.32$ & 4.40 & 6.36 & 1.10 & 4.36 & 1.00 & $-\infty$ \\
\hline $21 \ldots \ldots$ & $\mathrm{Sc}$ & 0.28 & $\lesssim-1.15$ & 0.77 & 3.10 & 0.46 & 0.77 & 0.46 & $-\infty$ \\
\hline $22 \ldots \ldots$ & $\mathrm{Ti}$ & 2.43 & $\lesssim 1.00$ & 2.87 & 4.99 & 0.76 & 2.99 & 1.00 & $-\infty$ \\
\hline $23 \ldots \ldots$ & $\mathrm{V}$ & 1.15 & $\lesssim-0.28$ & 1.87 & 4.00 & 0.74 & 2.00 & 1.00 & $-\infty$ \\
\hline $24 \ldots \ldots$ & $\mathrm{Cr}$ & 2.40 & $\lesssim 0.97$ & 3.17 & 5.67 & 0.32 & 3.17 & 0.32 & 5.50 \\
\hline $25 \ldots \ldots$ & $\mathrm{Mn}$ & 1.90 & $\lesssim 0.47$ & 2.56 & 5.39 & 0.15 & 2.56 & 0.15 & 5.32 \\
\hline $26 \ldots \ldots$ & $\mathrm{Fe}$ & 4.51 & $\lesssim 3.08$ & 5.03 & 7.51 & 0.33 & 5.03 & 0.33 & 7.34 \\
\hline $27 \ldots \ldots$ & Co & 2.24 & $\lesssim 0.81$ & 2.05 & 4.92 & 0.13 & 2.05 & 0.13 & 4.86 \\
\hline $28 \ldots \ldots$ & $\mathrm{Ni}$ & 3.25 & $\lesssim 1.82$ & 3.50 & 6.25 & 0.18 & 3.50 & 0.18 & 6.16 \\
\hline \multicolumn{10}{|c|}{ Group 3} \\
\hline $29 \ldots \ldots$ & $\mathrm{Cu}$ & 0.53 & $\lesssim-0.90$ & $?$ & 4.21 & $?$ & $?$ & $?$ & $?$ \\
\hline $30 \ldots \ldots$ & $\mathrm{Zn}$ & 1.86 & $\lesssim 0.43$ & $?$ & 4.60 & $?$ & $?$ & $?$ & $?$ \\
\hline $32 \ldots \ldots$ & $\mathrm{Ge}$ & $<-0.05$ & $?$ & $?$ & 3.41 & $?$ & $?$ & $?$ & $-\infty$ \\
\hline \multicolumn{10}{|c|}{ Group 4} \\
\hline $38 \ldots \ldots$ & $\mathrm{Sr}$ & 0.13 & -1.30 & 0.35 & 2.90 & 0.34 & 0.35 & 0.34 & $-\infty$ \\
\hline $56 \ldots \ldots$ & $\mathrm{Ba}$ & $\approx-1.80$ & -1.57 & -0.47 & 2.13 & 0.45 & -0.47 & 0.45 & $-\infty$ \\
\hline
\end{tabular}

$P$ INVEntory, SN II $(H)$ ANd II $(L)$ Yields, AND SN Ia Yield Pattern

NoTE.- Results are given for four groups of elements with the focus on groups 2 and 4 . The $P$ inventory in col. (3) and SN $\mathrm{II}(H)$ yields in col. (4) are calculated from the data shown in Fig. $2 a$ for groups $1-3$. The SN II $(L)$ yields in col. (5) are calculated from col. (3) and the data on HD 178443 (McWilliam et al. 1995) for group 2, and that for O is calculated by attributing the solar $\mathrm{O}$ inventory to $10^{2} \mathrm{SNe} \mathrm{II}(L)$. Col. (6) gives the solar inventory as measured in the photosphere (Anders \& Grevesse 1989). Col. (7) gives the fraction of the solar inventory of an element E contributed by SNe II as calculated from cols. (4)-(6). The corrected values in cols. (8) and (9) only reflect small adjustments for those elements with $\alpha_{\odot, \mathrm{II}}(\mathrm{E}) \approx 1$. The SN Ia yield pattern is represented by the part of the solar inventory contributed by SNe Ia in col. (10) as calculated from cols. (6) and (9). The results for group 4 are taken from QW01c but with $\log \epsilon_{P}(\mathrm{Ba})$ estimated from the data at $[\mathrm{Fe} / \mathrm{H}] \lesssim-3$ (see Fig. 1 ).

${ }^{\text {a }}$ Atomic number. 
data at $-4 \lesssim[\mathrm{Fe} / \mathrm{H}]<-3$. We use the results of Norris, Ryan, \& Beers $(2001)$ on $\mathrm{CD}-24^{\circ} 17504([\mathrm{Fe} / \mathrm{H}]=-3.37$; squares $), \mathrm{CD}-38^{\circ} 245([\mathrm{Fe} / \mathrm{H}]=-3.98 ;$ diamonds $), \mathrm{CS}$ $22172-002([\mathrm{Fe} / \mathrm{H}]=-3.61 ;$ triangles $)$, CS 22885-096 $([\mathrm{Fe} / \mathrm{H}]=-3.66 ;$ asterisks $)$, and $\mathrm{CS} 22949-037([\mathrm{Fe} / \mathrm{H}]=$ -3.79 ; plus signs). We have shifted the $\log \epsilon$ values shown for these five stars from the observed values to pass through $\log \epsilon(\mathrm{Fe})=\log \epsilon_{P}(\mathrm{Fe})=4.51$ so that they are compared at the same $\mathrm{Fe}$ abundance. It can be seen that the abundance patterns in these stars follow the $P$ inventory rather closely. Of these stars, CS 22949-037 exhibits the largest deviation from the $P$ inventory as it is overabundant in $\mathrm{Mg}$ and $\mathrm{Si}$. Data on this star from McWilliam et al. (1995) also show that it is overabundant in Na. However, the abundance pattern of all the other elements and the ratio $\mathrm{Si} / \mathrm{Mg}$ for this star are essentially the same as for the $P$ inventory. We thus consider that the $P$ inventory determined by the data at $[\mathrm{Fe} / \mathrm{H}] \approx-3$ is also an excellent representation of the data at $-4 \lesssim[\mathrm{Fe} / \mathrm{H}]<-3$. We infer that VMSs must therefore have a fairly uniform nucleosynthetic mechanism if they were responsible for the $P$ inventory. In $\S 3$ we will show that the $P$ inventory must also contain some contributions from $\mathrm{SNe} \operatorname{II}(H)$ and $\mathrm{II}(L)$.

\subsection{Estimates of $S N I I(L)$ Yields and SN I a Yield Pattern}

We now derive the $\mathrm{SN} \mathrm{II}(L)$ yields of $\mathrm{Na}$ to $\mathrm{Ni}$ based on the three-component model. By using the $\log \epsilon_{P}$ and $\log \epsilon_{H}$ values in Table 1 together with equation (1), the $\log \epsilon_{L}$ values of these elements can be obtained from the observed $\log \epsilon$ values for HD 178443 with $n_{H}=19.5$ and $n_{L}=2.45$ $([\mathrm{Fe} / \mathrm{H}]=-2.04 ; \mathrm{McWilliam}$ et al. 1995). The results are given in Table 1. It can be seen that typically $\log \epsilon_{L}(\mathrm{E})$ exceeds $\log \epsilon_{H}(\mathrm{E})$ by $\approx 2$ dex. Thus, although SNe II $(H)$ are 10 times more frequent than $\mathrm{SNe} \mathrm{II}(L)$ (e.g., QW01c), numerically the $\mathrm{SN} \operatorname{II}(H)$ contributions to the elements from $\mathrm{Na}$ to $\mathrm{Ni}$ can be ignored in general.

Using the $\mathrm{SN} \operatorname{II}(H)$ and $\operatorname{II}(L)$ yields, we give in Table 1 the fraction $\alpha_{\odot, \text { II }}(E)$ of the solar inventory of an element $E$ contributed by SNe II:

$$
\alpha_{\odot, \mathrm{II}}(\mathrm{E}) \equiv \frac{n_{H}^{\odot} \times 10^{\log \epsilon_{H}(\mathrm{E})}+n_{L}^{\odot} \times 10^{\log \epsilon_{L}(\mathrm{E})}}{10^{\log \epsilon_{\odot}(\mathrm{E})}}
$$

where $n_{H}^{\odot}=10^{3}$ and $n_{L}^{\odot}=10^{2}$ (QW01c) are the numbers of $\mathrm{SNe} \operatorname{II}(H)$ and $\operatorname{II}(L)$, respectively, that had occurred in a standard dilution mass of ISM prior to solar system formation (SSF). The contributions from the $P$ inventory to the solar inventory are negligible. Table 1 shows that $\mathrm{SNe} \mathrm{II}(L)$ can essentially account for the entire solar inventory of $\mathrm{Na}$, $\mathrm{Mg}, \mathrm{Si}, \mathrm{Ca}, \mathrm{Ti}$, and $\mathrm{V}\left[\alpha_{\odot, \mathrm{II}}(\mathrm{E}) \approx 1\right.$ for these elements as $\left.\log \epsilon_{L}(\mathrm{E})+\log n_{L}^{\odot} \approx \log \epsilon_{\odot}(\mathrm{E})\right]$. By contrast, Table 1 shows that the $\alpha_{\odot, \text { II }}$ values for Al, Sc, Cr, Mn, Fe, Co, and $\mathrm{Ni}$ only range from 0.13 to 0.47 . In view of the uncertainties in the data used to derive the $\log \epsilon_{L}$ values, we now choose to assign the entire solar inventory of $\mathrm{Na}, \mathrm{Mg}, \mathrm{Si}, \mathrm{Ca}, \mathrm{Ti}$, and $\mathrm{V}$ to $\mathrm{SNe} \operatorname{II}(L)$ [i.e., we take the "corrected" values of $\log \epsilon_{L}^{\text {corr }}(\mathrm{E})=\log \epsilon_{\odot}(\mathrm{E})-\log n_{L}^{\odot}$ and $\alpha_{\odot, \text { II }}^{\text {corr }}(\mathrm{E})=1$ for these elements]. For Al, Sc, Cr, Mn, Fe, Co, and Ni that have $\alpha_{\odot, \text { II }}$ values significantly below unity, we will use the original calculated values [i.e., $\log \epsilon_{L}^{\text {corr }}(E)=\log \epsilon_{L}(E)$ and $\alpha_{\odot, \mathrm{II}}^{\text {corr }}(\mathrm{E})=\alpha_{\odot, \mathrm{II}}(\mathrm{E})$ for these elements]. The $\mathrm{SN} \mathrm{II}(L)$ yields represented by the $\log \epsilon_{L}^{\text {corr }}$ values in Table 1 are shown in Figure 3 along with the solar abundance pattern that is translated to pass through $\log \epsilon_{L}^{\text {corr }}(\mathrm{Mg})$.

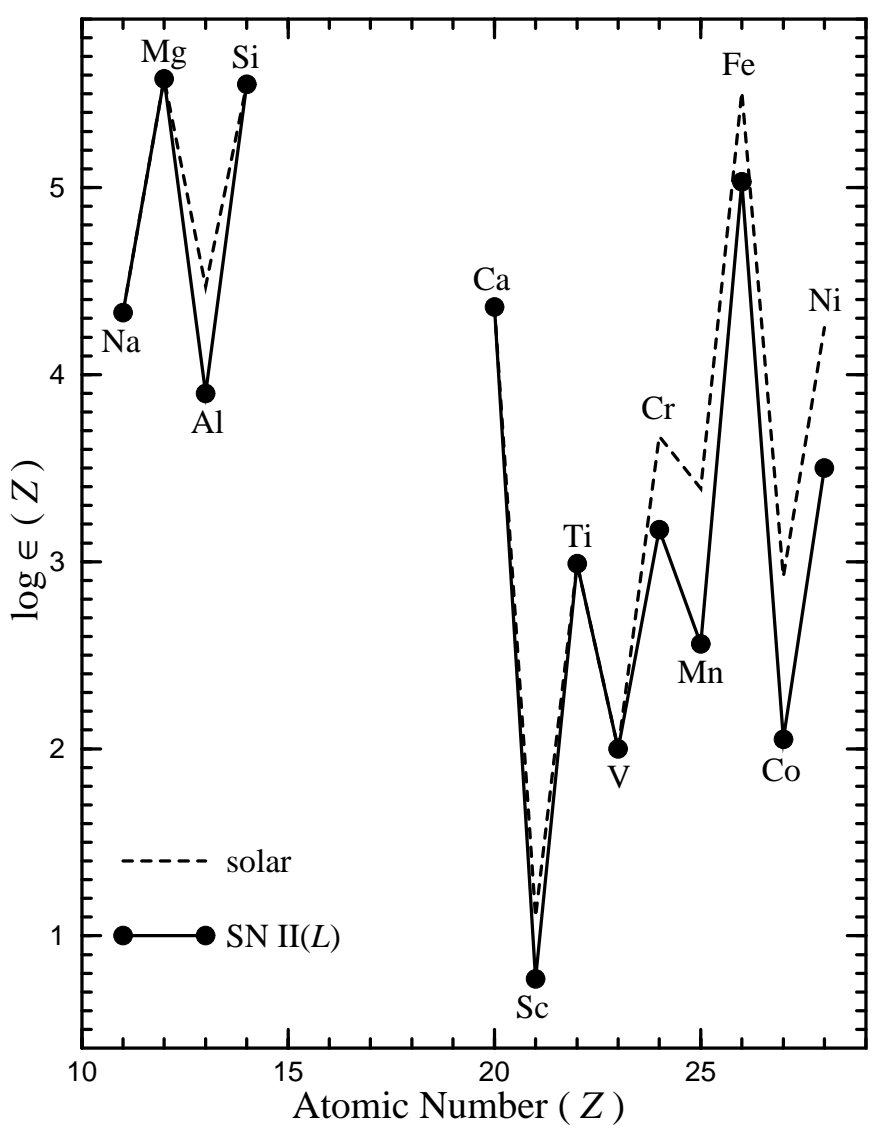

FIG. 3.- $\mathrm{SN} \mathrm{II}(L)$ yields ( filled circles connected with solid curves) calculated from the data on HD 178443 (McWilliam et al. 1995) by subtracting the small contributions from the $P$ inventory and $\mathrm{SNe} \mathrm{II}(H)$. The solar abundance pattern translated to pass through $\log \epsilon(\mathrm{Mg})=\log \epsilon_{L}(\mathrm{Mg})=$ 5.58 (dashed curve) is shown for comparison. With the exception of $\mathrm{Al}$ and $\mathrm{Sc}$, the translated solar abundance pattern up through $\mathrm{V}$ coincides with the $\mathrm{SN}$ II $(L)$ yields. The differences for the Fe group elements $(\mathrm{Cr}, \mathrm{Mn}, \mathrm{Fe}, \mathrm{Co}$, and $\mathrm{Ni}$ ) are attributed to the sum of SN Ia contributions corresponding to the $\log \epsilon_{\odot, \text { Ia }}$ values in Table 1 .

The majority of the solar inventory of the so-called $\mathrm{Fe}$ group elements ( $\mathrm{Cr}, \mathrm{Mn}, \mathrm{Fe}, \mathrm{Co}$, and $\mathrm{Ni}$ ) may be reasonably attributed to SNe Ia (e.g., Timmes, Woosley, \& Weaver 1995). In this case, the part of the solar inventory of an element $\mathrm{E}$ in the $\mathrm{Fe}$ group contributed by $\mathrm{SNe} \mathrm{Ia}$, $\log \epsilon_{\odot, \mathrm{Ia}}(\mathrm{E})$, can be estimated as

$$
\log \epsilon_{\odot, \mathrm{Ia}}(\mathrm{E})=\log \left[1-\alpha_{\odot, \mathrm{II}}(\mathrm{E})\right]+\log \epsilon_{\odot}(\mathrm{E}) .
$$

The $\log \epsilon_{\odot, \mathrm{Ia}}$ values for $\mathrm{Cr}, \mathrm{Mn}, \mathrm{Fe}, \mathrm{Co}$, and Ni estimated in equation (9) represent the yield pattern of these elements for $\mathrm{SNe}$ Ia and are given in Table 1.

\section{THE $P$ INVENTORY AND VMS YIELD PATTERN}

We have established the $P$ inventory and $\mathrm{SN} \mathrm{II}(H)$ and $\mathrm{II}(L)$ yields of $\mathrm{Na}$ to $\mathrm{Ni}$ in $\S 2$. We now focus on the $P$ inventory. This inventory was considered to be the result of the integrated production by VMSs over the period prior to the achievement of a metallicity of $[\mathrm{Fe} / \mathrm{H}] \approx-3$ in the ISM (WQ00a). Figure $2 b$ shows that the $P$ inventory is also an excellent representation of the abundance patterns at $-4 \lesssim[\mathrm{Fe} / \mathrm{H}]<-3$. This suggests that the yields of VMSs must follow a rather regular pattern. We will seek to establish the yield pattern of VMSs from the data at $-4 \lesssim[\mathrm{Fe} / \mathrm{H}] \lesssim-3$. It is important to recognize that very old stars with $-4 \lesssim[\mathrm{Fe} / \mathrm{H}]<-3$ must have low masses of 
TABLE 2

VMS YIELD PATTERNS

\begin{tabular}{|c|c|c|c|c|c|c|c|c|c|c|c|}
\hline \multirow[b]{2}{*}{$Z^{\mathrm{a}} \ldots \ldots$} & \multirow[b]{2}{*}{$\mathrm{E}$} & \multicolumn{2}{|c|}{$\mathrm{CD}-38^{\circ} 245$} & \multicolumn{2}{|c|}{ CS 22172-002 } & \multicolumn{2}{|c|}{ CS 22885-096 } & \multicolumn{2}{|c|}{ CS 22949-037 } & \multicolumn{2}{|c|}{$P$ INVENTORY } \\
\hline & & $f_{H L}(\mathrm{E})$ & $\log \epsilon_{\mathrm{VMS}}(\mathrm{E})$ & $f_{H L}(\mathrm{E})$ & $\log \epsilon_{\mathrm{VMS}}(\mathrm{E})$ & $f_{H L}(\mathrm{E})$ & $\log \epsilon_{\mathrm{VMS}}(\mathrm{E})$ & $f_{H L}(\mathrm{E})$ & $\log \epsilon_{\mathrm{VMS}}(\mathrm{E})$ & $f_{H L}(\mathrm{E})$ & $\log \epsilon_{\mathrm{VMS}}(\mathrm{E})$ \\
\hline $11 \ldots \ldots$ & $\mathrm{Na}$ & 0.42 & 3.53 & $\ldots$ & $\ldots$ & 0.14 & 3.56 & $<0.01$ & 5.87 & 0.26 & 3.77 \\
\hline $12 \ldots \ldots$ & $\mathrm{Mg}$ & 0.11 & 5.55 & 0.07 & 5.46 & 0.02 & 5.62 & 0.02 & 6.36 & 0.07 & 5.66 \\
\hline $13 \ldots \ldots$ & $\mathrm{Al}$ & 0.49 & 2.97 & 0.36 & 2.88 & 0.13 & 3.16 & 0.23 & 3.49 & 0.16 & 3.60 \\
\hline $14 \ldots \ldots$ & $\mathrm{Si}$ & 0.15 & 5.34 & 0.06 & 5.44 & 0.03 & 5.51 & 0.03 & 6.15 & 0.09 & 5.54 \\
\hline $20 \ldots \ldots$ & $\mathrm{Ca}$ & 0.22 & 3.96 & 0.11 & 3.99 & 0.04 & 4.15 & 0.11 & 4.33 & 0.11 & 4.26 \\
\hline $21 \ldots \ldots$ & $\mathrm{Sc}$ & 0.12 & 0.69 & 0.07 & 0.64 & 0.03 & 0.77 & 0.11 & 0.75 & 0.08 & 0.80 \\
\hline $22 \ldots \ldots$ & $\mathrm{Ti}$ & 0.14 & 2.82 & 0.08 & 2.80 & 0.05 & 2.67 & 0.14 & 2.84 & 0.10 & 2.95 \\
\hline $23 \ldots \ldots$ & V & $>0.09$ & $<2.04$ & $\ldots$ & $\ldots$ & $>0.04$ & $<1.82$ & $>0.07$ & $<2.19$ & 0.19 & 1.62 \\
\hline $24 \ldots \ldots$ & $\mathrm{Cr}$ & 0.44 & 2.34 & 0.21 & 2.48 & 0.10 & 2.57 & 0.36 & 2.49 & 0.15 & 2.89 \\
\hline $25 \ldots \ldots$ & $\mathrm{Mn}$ & 0.51 & 1.59 & 0.29 & 1.69 & 0.07 & 2.10 & 0.40 & 1.82 & 0.12 & 2.40 \\
\hline $26 \ldots \ldots$ & $\mathrm{Fe}$ & 0.10 & 5.03 & 0.05 & 5.03 & 0.03 & 5.03 & 0.11 & 5.03 & 0.09 & 5.03 \\
\hline $27 \ldots \ldots$ & Co & 0.01 & 2.97 & 0.01 & 3.03 & $<0.01$ & 3.04 & 0.01 & 3.06 & 0.02 & 2.79 \\
\hline $28 \ldots \ldots$ & $\mathrm{Ni}$ & 0.07 & 3.66 & 0.03 & 3.80 & 0.01 & 3.96 & 0.05 & 3.86 & 0.05 & 3.79 \\
\hline $38 \ldots \ldots$ & $\mathrm{Sr}$ & 0.54 & -0.57 & 0.85 & -1.58 & 1.10 & $-\infty$ & 0.09 & 0.53 & 0.05 & 0.67 \\
\hline
\end{tabular}

Note. - Results are calculated from the data on the four listed stars with $[\mathrm{Fe} / \mathrm{H}]<-3$ (McWilliam et al. 1995 for Na; Norris et al. 2001 for all the other elements) and from the $P$ inventory as determined for $[\mathrm{Fe} / \mathrm{H}]=-3$. The parameter $f_{H L}(\mathrm{E})$ is the fraction of an element $\mathrm{E}$ in a star or in the $P$ inventory contributed by $\mathrm{SNe} \mathrm{II}(H)$ and $\mathrm{II}(L)$. The VMS yield pattern is represented by $\log \epsilon_{\mathrm{VMS}}(\mathrm{E})$ as calculated from $f_{H L}(\mathrm{E})$ and the observed log $\epsilon(\mathrm{E})$ in a star or $\log \epsilon_{P}(\mathrm{E})$. For convenience of comparison, the $\log \epsilon_{\mathrm{VMS}}$ values have been shifted to pass through the same Fe abundance of $\log \epsilon_{\mathrm{VMS}}(\mathrm{Fe})=$ $\log \epsilon_{L}(\mathrm{Fe})=5.03$.

a Atomic number.

$\sim 1 M_{\odot}$ to have survived until the present time. This requires that there be some formation of stars in the normal mass range of $\sim 1-60 M_{\odot}$ in addition to the formation of VMSs with masses of $\gtrsim 100 M_{\odot}$ considerably before a metallicity of $[\mathrm{Fe} / \mathrm{H}] \approx-3$ was reached in the ISM. As normal stars with masses of $\sim 10-60 M_{\odot}$ are SN II progenitors, we consider that $\mathrm{SNe} \mathrm{II}(H)$ and $\mathrm{II}(L)$ must have provided chemical enrichment of the ISM along with VMSs at $-4 \lesssim[\mathrm{Fe} / \mathrm{H}]<-3$. Thus, the $P$ inventory at $[\mathrm{Fe} / \mathrm{H}]=$ -3 and the abundances in stars with $-4 \lesssim[\mathrm{Fe} / \mathrm{H}]<-3$ should be the sum of contributions from VMSs and SNe II $(H)$ and $\operatorname{II}(L)$. The yield pattern of VMSs must then be obtained by subtracting the $\mathrm{SN} \mathrm{II}(H)$ and $\mathrm{II}(L)$ contributions from the $P$ inventory or the observed abundances at $-4 \lesssim[\mathrm{Fe} / \mathrm{H}]<-3$. This can be accomplished by using the observed $\mathrm{Ba}$ abundances. The observed $\mathrm{Ba}$ abundances at $-4 \lesssim[\mathrm{Fe} / \mathrm{H}]<-3$ are particularly important as they also are the key evidence requiring a much larger dilution mass in the regime where VMSs are active in comparison with the regime of $[\mathrm{Fe} / \mathrm{H}] \gtrsim-3$ where VMSs are no longer active.

Unlike SNe II, VMSs explode as a result of pair instability (e.g., Rakavy, Shaviv, \& Zinamon 1967; Bond, Arnett, \& Carr 1984; Glatzel, Fricke, \& El Eid 1985; Heger \& Woosley 2002). We do not consider VMSs as a source of $r$-process elements such as $\mathrm{Ba}$. On the other hand, small abundances of $\mathrm{Ba}$ have been observed at $-4 \lesssim[\mathrm{Fe} / \mathrm{H}]<-3$ (e.g., McWilliam et al. 1995; McWilliam 1998; Norris et al. 2001). This was previously considered by us to be the result of contamination of the VMS-enriched ISM by a small amount of SN II $(H)$ ejecta that contains Ba but very little Fe (WQ00a). We now consider that some level of "normal" astration was present in the regime where VMSs were active and that the abundances in the ISM at $-4 \lesssim[\mathrm{Fe} / \mathrm{H}]<-3$ consist of contributions from VMSs and $\mathrm{SNe} \operatorname{II}(H)$ and $\mathrm{II}(L)$. As Ba is not considered to be produced by VMSs, we may attribute the $\mathrm{Ba}$ in a star with these metallicities to an effective number $\tilde{n}_{H}$ of $\mathrm{SNe} \mathrm{II}(H)$ and an effective number $\tilde{n}_{L}$ of $\mathrm{SNe} \mathrm{II}(L)$ that had contributed to the ISM from which the star was formed:

$$
10^{\log \epsilon(\mathrm{Ba})}=\tilde{n}_{H} \times 10^{\log \epsilon_{H}(\mathrm{Ba})}+\tilde{n}_{L} \times 10^{\log \epsilon_{L}(\mathrm{Ba})},
$$

where $\log \epsilon_{H}(\mathrm{Ba})=-1.57$ or $\log \epsilon_{L}(\mathrm{Ba})=-0.47$ corresponds to the abundance of $\mathrm{Ba}$ in the ISM resulting from a single $\mathrm{SN} \operatorname{II}(H)$ or $\mathrm{II}(L)$ for a standard dilution mass of $M_{\mathrm{dil}}^{\mathrm{SNII}} \approx 3 \times 10^{4} M_{\odot} \quad(\mathrm{QW} 01 \mathrm{c})$. The lowest observed $\log \epsilon(\mathrm{Ba})$ value is -2.97 for $\mathrm{CS} 22885-096([\mathrm{Fe} / \mathrm{H}]=$ -3.66 ; Norris et al. 2001). Equation (10) gives $\tilde{n}_{H}=0.04$ if the $\mathrm{Ba}$ abundance in this star was provided by a single SN II $(H)$ or $\tilde{n}_{L}=3.16 \times 10^{-3}$ for a single SN II $(L)$. As the SN II $(H$ and II $(L$ yields are fixed, we consider that these low values of $\tilde{n}_{H}$ and $\tilde{n}_{L}$ (which should be approximately integers for an appropriate dilution mass) require a much larger dilution mass for the SN II ejecta at $-4 \lesssim[\mathrm{Fe} / \mathrm{H}]<-3$ compared with the standard dilution mass at $[\mathrm{Fe} / \mathrm{H}] \gtrsim$ -3 . This is in accord with the extremely energetic explosions of VMSs (e.g., Heger \& Woosley 2002) that would occur along with $\mathrm{SNe}$ II at $-4 \lesssim[\mathrm{Fe} / \mathrm{H}]<-3$. Specifically, the dilution mass $M_{\mathrm{dil}}^{\mathrm{VMS}}$ relevant for these ultralow metallicities is at least $M_{\mathrm{dil}}^{\mathrm{SNII}} / 0.04 \approx 8 \times 10^{5} M_{\odot}$ and may be as large as $M_{\mathrm{dil}}^{\mathrm{SNII}} /\left(3.16 \times 10^{-3}\right) \approx 9 \times 10^{6} M_{\odot}$ (the difference in the dilution masses for $[\mathrm{Fe} / \mathrm{H}]<-3$ and $[\mathrm{Fe} / \mathrm{H}] \gtrsim-3$ was considered earlier by Qian \& Wasserburg 2001a in discussing the $P$ inventory of $O$ ).

We now use the $\mathrm{Ba}$ abundances to estimate the SN II contributions for stars with $-4 \lesssim[\mathrm{Fe} / \mathrm{H}]<-3$ in order to obtain the "pure" VMS contributions. As explosions of VMSs can disrupt regions with a mass much larger than the standard dilution mass for SNe II, we assume that the overall mixing occurs over the region governed by VMS explosions. We also assume that $\tilde{n}_{H} / \tilde{n}_{L}=10$ for a star with $-4 \lesssim[\mathrm{Fe} / \mathrm{H}]<-3$ and use equation (10) to estimate $\tilde{n}_{L}$ as

$$
\tilde{n}_{L}=10^{\log \epsilon(\mathrm{Ba})-\log \epsilon_{H L}(\mathrm{Ba})}=10^{\log \epsilon(\mathrm{Ba})+0.22},
$$

where we have defined

$$
10^{\log \epsilon_{H L}(\mathrm{Ba})} \equiv 10 \times 10^{\log \epsilon_{H}(\mathrm{Ba})}+10^{\log \epsilon_{L}(\mathrm{Ba})}=10^{0.22} .
$$




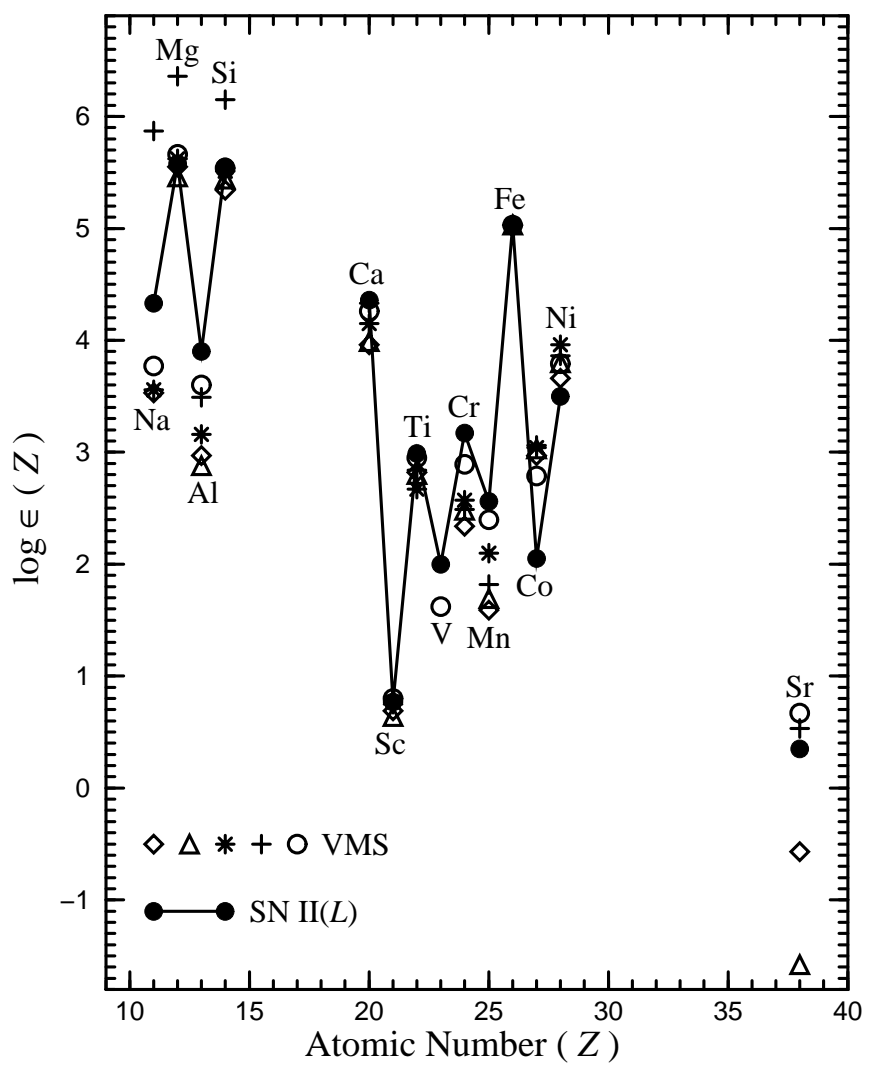

FIG. 4.-VMS yield patterns calculated from the data on four stars with $[\mathrm{Fe} / \mathrm{H}]<-3$ (diamonds: $\mathrm{CD}-38^{\circ} 245$; triangles: CS 22172-002; asterisks: CS 22885-096; plus signs: CS 22949-037; McWilliam et al. 1995 for $\mathrm{Na}$ and Norris et al. 2001 for all the other elements) and that calculated from the $P$ inventory at $[\mathrm{Fe} / \mathrm{H}]=-3$ (open circles) by using $\mathrm{Ba}$ as the index of SN II production. These patterns have been translated to pass through the same Fe abundance of $\log \epsilon(\mathrm{Fe})=\log \epsilon_{L}(\mathrm{Fe})=5.03$ for comparison with the $\mathrm{SN} \mathrm{II}(L)$ yield pattern (filled circles connected by solid curves). Note the general similarity between the VMS and SN II $(L)$ yield patterns. However, VMSs show some high production of $\mathrm{Na}, \mathrm{Mg}$, and $\mathrm{Si}$ from the data on one star (plus signs: CS 22949-037), a low production of $\mathrm{Na}$ from the data on the other stars, and in all cases a significantly lower production of $\mathrm{Al}, \mathrm{V}, \mathrm{Cr}$, and $\mathrm{Mn}$ and a significantly higher production of Co. Except for CS 22885-096 (asterisks with no $\mathrm{Sr}$ assignment), the $\mathrm{Sr}$ in all the other three stars and in the $P$ inventory is not explained by SN II coproduction with $\mathrm{Ba}$. This is considered as a strong hint of $\mathrm{Sr}$ production by some VMSs.

The results presented below are not at all sensitive to the choice of $\tilde{n}_{H} / \tilde{n}_{L}=10$ as the Ba yield of $\mathrm{SNe} \mathrm{II}(L)$ is $\approx 13$ times larger than that of $\mathrm{SNe} \mathrm{II}(H)$ (see Table 1). For an element $\mathrm{E}$ other than $\mathrm{Ba}$, the fraction $f_{H L}(\mathrm{E})$ of this element in the star contributed by $\mathrm{SNe} \operatorname{II}(H)$ and $\mathrm{II}(L)$ is

$$
f_{H L}(\mathrm{E}) \equiv \tilde{n}_{L} \times 10^{\log \epsilon_{H L}(\mathrm{E})-\log \epsilon(\mathrm{E})},
$$

where $\log \epsilon_{H L}(\mathrm{E})$ is defined in a similar manner to $\log \epsilon_{H L}(\mathrm{Ba})$ and can be calculated from the $\log \epsilon_{H}$ and $\log \epsilon_{L}^{\text {corr }}$ values in Table 1 . The remaining fraction $\left[1-f_{H L}(\mathrm{E})\right]$ is then attributed to VMSs:

$$
10^{\log \epsilon \operatorname{VMS}(\mathrm{E})}=\left[1-f_{H L}(\mathrm{E})\right] \times 10^{\log \epsilon(\mathrm{E})},
$$

where $\log \epsilon_{\mathrm{VMS}}(\mathrm{E})$ represents the total VMS contributions to the element $E$ in the star. The above equation also applies to the $P$ inventory.

By using the $\log \epsilon_{H}$ and $\log \epsilon_{L}^{\text {corr }}$ values in Table 1 together with equations (11), (13), and (14), the $f_{H L}$ and $\log \epsilon_{\mathrm{VMS}}$ values for $\mathrm{Na}$ to $\mathrm{Ni}$ and $\mathrm{Sr}$ are calculated from the data on four stars with $[\mathrm{Fe} / \mathrm{H}]=-3.98$ to -3.61 (Norris et al. 2001) and from the $P$ inventory as determined at $[\mathrm{Fe} / \mathrm{H}]=-3$. These results are given in Table 2. The VMS yield patterns represented by the $\log \epsilon_{\mathrm{VMS}}$ values are also shown in Figure 4. For convenience of comparison, the $\log \epsilon_{\mathrm{VMS}}$ values given in Table 2 and shown in Figure 4 have been shifted from the original calculated values to pass through the same $\mathrm{Fe}$ abundance of $\log \epsilon_{\mathrm{VMS}}(\mathrm{Fe})=$ $\log \epsilon_{L}(\mathrm{Fe})=5.03$. It can be seen that the yield patterns of VMSs derived from the four stars and that derived from the $P$ inventory are almost identical to each other. The only exceptions are the anomalies at $\mathrm{Na}, \mathrm{Mg}$, and $\mathrm{Si}$ in $\mathrm{CS}$ 22949-037 as noted earlier and the varying production of $\mathrm{Sr}$. Table 2 also shows that VMSs dominated the production of $\mathrm{Na}$ to $\mathrm{Ni}$ at $-4 \lesssim[\mathrm{Fe} / \mathrm{H}] \lesssim-3$ and that $\mathrm{SNe} \mathrm{II}(L)$ contributed $\sim 3 \%-10 \%$ of the $\mathrm{Fe}$ at these metallicities.

The VMS yield patterns derived above may be compared with the SN II $(L)$ yield pattern. Figure 4 shows that relative to $\mathrm{SNe} \mathrm{II}(L)$, VMSs underproduce $\mathrm{Na}, \mathrm{Al}, \mathrm{V}, \mathrm{Cr}$, and $\mathrm{Mn}$, overproduce $\mathrm{Co}$, produce a variable amount of $\mathrm{Sr}$, but otherwise have an almost identical yield pattern. The $\mathrm{Sr}$ production by VMSs is small or completely negligible based on the data for CD - 38 245 , CS 22172-002, and CS 22885096 but may be somewhat larger than that by $\mathrm{SNe} \operatorname{II}(L)$ based on the data for CS 22949-037 and the $P$ inventory. Thus, there appears to be a strong hint for major Sr production by some VMSs.

\section{MODEL FOR GENERAL CHEMICAL EVOLUTION}

We have presented in $\S \S 2$ and 3 the results on $\mathrm{SN} \mathrm{II}(H)$ and $\operatorname{II}(L)$ yields and SN Ia and VMS yield patterns, all of which appear to be rather well defined. Based on these results, we now discuss a model for general chemical evolution of the ISM starting from a zero-metallicity state. We will extend the earlier approach for $[\mathrm{Fe} / \mathrm{H}] \gtrsim-3$ (e.g., Qian \& Wasserburg 2001a; QW01c) to $[\mathrm{Fe} / \mathrm{H}]<-3$. The production by VMSs and $\mathrm{SNe} \mathrm{II}(H), \mathrm{II}(L)$, and Ia, as well as the difference in the dilution masses associated with SNe II and VMSs, will be taken into account. A full approach must address the discreteness of the various production events. We will first use a continuous approximation to illustrate some essential features of the model and discuss the effects of discrete events later. We use $\mathrm{E}$ to represent an element other than $\mathrm{Fe}$. We consider the following sequence of evolution for the abundances of $\mathrm{E}$ and $\mathrm{Fe}$ in the ISM. Starting from big bang debris with zero metallicity, i.e., $\log \epsilon(\mathrm{E})=-\infty$ and $[\mathrm{Fe} / \mathrm{H}]=-\infty$, the ISM was first enriched in $\mathrm{E}$ and $\mathrm{Fe}$ by VMSs and $\mathrm{SNe} \mathrm{II}(H)$ and $\mathrm{II}(L)$. When a metallicity of $[\mathrm{Fe} / \mathrm{H}] \approx-3$ was reached, VMSs ceased occurring. At $-3 \lesssim[\mathrm{Fe} / \mathrm{H}]<-1$, only $\mathrm{SNe} \mathrm{II}(H)$ and $\operatorname{II}(L)$ provided chemical enrichment of the ISM, while at $[\mathrm{Fe} / \mathrm{H}] \gtrsim-1, \mathrm{SNe} \mathrm{II}(H), \mathrm{II}(L)$, and Ia all contributed. We are concerned here only with the contributions from VMSs and SNe II and Ia, but not with the contributions from low-mass stars such as asymptotic giant branch (AGB) stars. AGB stars would make $s$-process contributions to the heavy elements above $\mathrm{Fe}$ at $[\mathrm{Fe} / \mathrm{H}] \gtrsim-1$. Thus, we restrict our discussion to the elements from $\mathrm{Na}$ to $\mathrm{Ni}$ for $[\mathrm{Fe} / \mathrm{H}] \leq 0$ and to the $r$-process elements ( $\mathrm{Sr}$ and above) for $[\mathrm{Fe} / \mathrm{H}]<-1$.

\subsection{Continuous Model without SN Ia Contributions}

We here consider a continuous model for evolution of elemental abundances. This model is not applicable in regions where the number of nucleosynthetic events is small 
and addition from an individual event causes significant increment in the inventory of an element. This approach does provide a good overall view and in particular shows quantitatively that, for example, the evolution of Ba relative to Fe with a jump at $[\mathrm{Fe} / \mathrm{H}] \approx-3$ is the result of the change from $\mathrm{Fe}$ production predominantly by VMSs to $\mathrm{Fe}$ production by $\mathrm{SNe} \mathrm{II}(L)$. The continuous model can be discussed without knowing the dilution masses for $[\mathrm{Fe} / \mathrm{H}]<-3$ and $[\mathrm{Fe} / \mathrm{H}] \gtrsim-3$. However, as we have already demonstrated the change in the dilution mass for these two regimes in $\S 3$, we will incorporate this behavior in the treatment here. The conclusion regarding the jump in the evolution of $\mathrm{Ba}$ does not depend on the different dilution masses for $[\mathrm{Fe} / \mathrm{H}]<-3$ and $[\mathrm{Fe} / \mathrm{H}] \gtrsim-3$.

We first discuss the regime of $[\mathrm{Fe} / \mathrm{H}]<-1$ that excludes SN Ia contributions. Consider a homogeneous system of gas with a time-dependent total number $(\mathrm{H})$ of $\mathrm{H}$ atoms. In the continuous approximation, the rate of change in the abundance of $\mathrm{E}$ in this gas, $d(\mathrm{E} / \mathrm{H}) / d t$, is $(\mathrm{QW} 01 \mathrm{c})$

$$
\frac{d(\mathrm{E} / \mathrm{H})}{d t}=\frac{P_{\mathrm{E}}^{\mathrm{VMS}}(t)+P_{\mathrm{E}}^{H}(t)+P_{\mathrm{E}}^{L}(t)}{(\mathrm{H})},
$$

where $P_{\mathrm{E}}^{\mathrm{VMS}}(t), P_{\mathrm{E}}^{H}(t)$, and $P_{\mathrm{E}}^{L}(t)$ are the rates for production of $\mathrm{E}$ by VMSs and $\mathrm{SNe} \mathrm{II}(H)$ and $\mathrm{II}(L)$, respectively. The production rates of individual sources are proportional to the absolute yields and the frequencies of these sources. Thus, the terms such as $P_{\mathrm{E}}^{\mathrm{VMS}}(t) /(\mathrm{H})$ represent the products of the absolute yields of each event and the frequency of occurrences per $\mathrm{H}$ atom in the ISM for the individual sources. We consider that the absolute yields of VMSs and $\mathrm{SNe} \mathrm{II}(H)$ and $\mathrm{II}(L)$ are fixed. We further assume that the frequencies of these sources are proportional to the total number $(\mathrm{H})$ of $\mathrm{H}$ atoms in the gas but with different proportionality constants for $0 \leq t<t_{*}$ and $t \geq t_{*}$, where $t_{*}$ is the time when $[\mathrm{Fe} / \mathrm{H}]=-3$ was reached. Then the production rates per $\mathrm{H}$ atom, denoted by $\widetilde{\mathscr{P}}_{\mathrm{E}}^{\mathrm{VMS}}, \tilde{\mathscr{P}}_{\mathrm{E}}^{H}$, and $\tilde{\mathscr{P}}_{\mathrm{E}}^{L}$ for $0 \leq t<t_{*}$ and by $\mathscr{P}_{\mathrm{E}}^{H}$ and $\mathscr{P}_{\mathrm{E}}^{L}$ for $t \geq t_{*}$, are all constant. We use a similar notation for Fe. Note that $\widetilde{P}_{\mathrm{Fe}}^{H}=\mathscr{P}_{\mathrm{Fe}}^{H}=0$ as $\mathrm{SNe} \mathrm{II}(H)$ produce no $\mathrm{Fe}$ (WQ00a). The production rates per $\mathrm{H}$ atom for $\mathrm{SNe} \mathrm{II}(H)$ and $\mathrm{II}(L)$ at $0 \leq t<t_{*}$ (with the tilde symbol) are related to those at $t \geq t_{*}$ (without the tilde symbol) by the very different dilution masses with which the SN II ejecta are mixed in these two epochs:

$$
\frac{\tilde{\mathscr{P}}_{\mathrm{E}}^{H}}{\mathscr{P}_{\mathrm{E}}^{H}}=\frac{\widetilde{\mathscr{P}}_{\mathrm{E}}^{L}}{\mathscr{P}_{\mathrm{E}}^{L}}=\frac{\tilde{\mathscr{P}}_{\mathrm{Fe}}^{L}}{\mathscr{P}_{\mathrm{Fe}}^{L}}=\frac{M_{\mathrm{dil}}^{\mathrm{SNII}}}{M_{\mathrm{dil}}^{\mathrm{VMS}}} .
$$

It is found in $\S 3$ that the dilution mass $M_{\mathrm{dil}}^{\mathrm{VMS}}$ for $0 \leq t<t_{*}$ is larger than the dilution mass $M_{\mathrm{dil}}^{\mathrm{SNII}}$ for $t \geq t_{*}$ by a factor of $\sim 25-300$.

With the notation introduced above, the rates of change in the abundances of $\mathrm{E}$ and $\mathrm{Fe}$ in the gas are

$$
\frac{d(\mathrm{E} / \mathrm{H})}{d t}= \begin{cases}\tilde{P}_{\mathrm{E}}^{\mathrm{VMS}}+\tilde{\mathscr{P}}_{\mathrm{E}}^{H}+\tilde{\mathscr{P}}_{\mathrm{E}}^{L}, & 0 \leq t<t_{*} \\ \mathscr{P}_{\mathrm{E}}^{H}+\mathscr{P}_{\mathrm{E}}^{L}, & t \geq t_{*}\end{cases}
$$

and

$$
\frac{d(\mathrm{Fe} / \mathrm{H})}{d t}= \begin{cases}\tilde{\mathscr{P}}_{\mathrm{Fe}}^{\mathrm{Ve}}+\tilde{\mathscr{P}}_{\mathrm{Fe}}^{L}, & 0 \leq t<t_{*} \\ \mathscr{P P}_{\mathrm{Fe}}^{L}, & t \geq t_{*}\end{cases}
$$

Eliminating $t$ in equations (17) and (18), we obtain

$$
\frac{d(\mathrm{E} / \mathrm{H})}{d(\mathrm{Fe} / \mathrm{H})}= \begin{cases}\frac{\widetilde{\mathscr{P}}_{\mathrm{E}}^{\mathrm{VMS}}+\tilde{\mathscr{P}}_{\mathrm{E}}^{H}+\tilde{\mathscr{P}}_{\mathrm{E}}^{L}}{\widetilde{\mathscr{P}}_{\mathrm{Fe}}^{\mathrm{VMS}}+\tilde{\mathscr{P}}_{\mathrm{Fe}}^{L}}, & {\left[\frac{\mathrm{Fe}}{\mathrm{H}}\right]<-3,} \\ \frac{\mathscr{P}_{\mathrm{E}}^{H}+\mathscr{P}_{\mathrm{E}}^{L}}{\mathscr{P}_{\mathrm{Fe}}^{L}}, & -3 \leq\left[\frac{\mathrm{Fe}}{\mathrm{H}}\right]<-1 .\end{cases}
$$

Equation (19) with the initial conditions $(\mathrm{E} / \mathrm{H})=$ $(\mathrm{Fe} / \mathrm{H})=0$ at $t=0$ can be solved to give

$$
\begin{aligned}
\left(\frac{\mathrm{E}}{\mathrm{H}}\right) & = \\
& \left\{\begin{aligned}
\left(\frac{\widetilde{\mathscr{P}}_{\mathrm{E}}^{\mathrm{VMS}}+\widetilde{\mathscr{P}}_{\mathrm{E}}^{H}+\tilde{\mathscr{P}}_{\mathrm{E}}^{L}}{\widetilde{\mathscr{P}}_{\mathrm{Fe}}^{\mathrm{VMS}}+\tilde{\mathscr{P}}_{\mathrm{Fe}}^{L}}\right)\left(\frac{\mathrm{Fe}}{\mathrm{H}}\right), & {\left[\frac{\mathrm{Fe}}{\mathrm{H}}\right]_{<-3,} } \\
\left(\frac{\mathrm{E}}{\mathrm{H}}\right)_{*}+\left(\frac{\mathscr{P P}_{\mathrm{E}}^{H}+\mathscr{P}_{\mathrm{E}}^{L}}{\mathscr{P P}_{\mathrm{Fe}}^{L}}\right)\left[\left(\frac{\mathrm{Fe}}{\mathrm{H}}\right)-\left(\frac{\mathrm{Fe}}{\mathrm{H}}\right)_{*}\right], & \\
& -3 \leq\left[\frac{\mathrm{Fe}}{\mathrm{H}}\right]<-1,
\end{aligned}\right.
\end{aligned}
$$

where $(\mathrm{E} / \mathrm{H})_{*}$ and $(\mathrm{Fe} / \mathrm{H})_{*}$ correspond to the abundances of $\mathrm{E}$ and $\mathrm{Fe}$ at $[\mathrm{Fe} / \mathrm{H}]=-3$.

The result for $[\mathrm{Fe} / \mathrm{H}]<-3$ in equation (20) can be rewritten as

$$
\log \epsilon(\mathrm{E})=\left[\frac{\mathrm{Fe}}{\mathrm{H}}\right]+\log \epsilon_{\odot}(\mathrm{Fe})+\log \left(\frac{\widetilde{\mathscr{P}}_{\mathrm{E}}^{\mathrm{VMS}}+\tilde{\mathscr{P}}_{\mathrm{E}}^{H}+\widetilde{\mathscr{P}}_{\mathrm{E}}^{L}}{\widetilde{\mathscr{P}}_{\mathrm{Fe}}^{\mathrm{VMS}}+\widetilde{\mathscr{P}}_{\mathrm{Fe}}^{L}}\right) .
$$

Thus, in the continuous approximation, the evolution of $\mathrm{E}$ relative to $\mathrm{Fe}$ at $[\mathrm{Fe} / \mathrm{H}]<-3$ follows a straight line of unit slope on a plot of $\log \epsilon(\mathrm{E})$ as a function of $[\mathrm{Fe} / \mathrm{H}]$. The position of this line on the plot is determined by the ratio of the relevant total production rates per $\mathrm{H}$ atom for $\mathrm{E}$ and $\mathrm{Fe}$. The result for $-3 \leq[\mathrm{Fe} / \mathrm{H}]<-1$ in equation (20) in the limit of $(\mathrm{E} / \mathrm{H}) \gg(\mathrm{E} / \mathrm{H})_{*}$ and $(\mathrm{Fe} / \mathrm{H}) \gg(\mathrm{Fe} / \mathrm{H})_{*}$ can be approximated as

$$
\begin{aligned}
\log \epsilon(\mathrm{E}) & \approx\left[\frac{\mathrm{Fe}}{\mathrm{H}}\right]+\log \epsilon_{\odot}(\mathrm{Fe})+\log \left(\frac{\mathscr{P}_{\mathrm{E}}^{H}+\mathscr{P}_{\mathrm{E}}^{L}}{\mathscr{P}_{\mathrm{Fe}}^{L}}\right) \\
& \approx\left[\frac{\mathrm{Fe}}{\mathrm{H}}\right]+\log \epsilon_{\odot}(\mathrm{Fe})+\log \left(\frac{\widetilde{P}_{\mathrm{E}}^{H}+\widetilde{\mathscr{P}}_{\mathrm{E}}^{L}}{\widetilde{\mathscr{P}}_{\mathrm{Fe}}^{L}}\right),
\end{aligned}
$$

where equation (16) is used in writing the last term. This again represents a straight line of unit slope. However, as a result of the termination of VMS activities at $[\mathrm{Fe} / \mathrm{H}]=-3$ and the corresponding change in the ratio of the total production rates per $\mathrm{H}$ atom for $\mathrm{E}$ and $\mathrm{Fe}$, the line for equation (22) is offset from that for equation (21) by

$$
\log \left[\left(\frac{\tilde{\mathscr{P}}_{\mathrm{E}}^{H}+\tilde{\mathscr{P}}_{\mathrm{E}}^{L}}{\tilde{\mathscr{P}}_{\mathrm{E}}^{\mathrm{VMS}}+\tilde{\mathscr{P}}_{\mathrm{E}}^{H}+\tilde{\mathscr{P}}_{\mathrm{E}}^{L}}\right)\left(\frac{\tilde{\mathscr{P}}_{\mathrm{Fe}}^{\mathrm{VM}}+\tilde{\mathscr{P}}_{\mathrm{Fe}}^{L}}{\tilde{\mathscr{P}}_{\mathrm{Fe}}^{L}}\right)\right] .
$$

Thus, the full evolutionary trajectory of $\log \epsilon(\mathrm{E})$ follows a straight line of unit slope up to $[\mathrm{Fe} / \mathrm{H}]=-3$ and then asymptotically approaches another straight line of unit slope. The sharpness of the transition from the initial line to the asymptotic line can be assessed by the slope of the 
evolutionary trajectory at $[\mathrm{Fe} / \mathrm{H}]=-3$ :

$$
\begin{aligned}
\left.\frac{d \log \epsilon(\mathrm{E})}{d[\mathrm{Fe} / \mathrm{H}]}\right|_{*} & =\left.\frac{d(\mathrm{E} / \mathrm{H})}{d(\mathrm{Fe} / \mathrm{H})}\right|_{*} \frac{(\mathrm{Fe} / \mathrm{H})_{*}}{(\mathrm{E} / \mathrm{H})_{*}} \\
& =\left(\frac{\tilde{P}_{\mathrm{E}}^{H}+\tilde{\mathscr{P}}_{\mathrm{E}}^{L}}{\tilde{\mathscr{P}}_{\mathrm{E}}^{\mathrm{VMS}}+\tilde{\mathscr{P}}_{\mathrm{E}}^{H}+\tilde{\mathscr{P}}_{\mathrm{E}}^{L}}\right)\left(\frac{\tilde{P}_{\mathrm{Fe}}^{\mathrm{VMS}}+\tilde{\mathscr{P}}_{\mathrm{Fe}}^{L}}{\tilde{\mathscr{P}}_{\mathrm{Fe}}^{L}}\right) .
\end{aligned}
$$

Note that $\tilde{P}_{\mathrm{E}}^{\mathrm{VMS}}=0$ for $r$-process elements such as Ba. For these elements the shift in equation $(23)$ is $\log \left[\left(\tilde{\mathscr{P}}_{\mathrm{Fe}}^{\mathrm{VMS}}\right.\right.$ $\left.\left.+\tilde{\mathscr{P}}_{\mathrm{Fe}}^{L}\right) / \tilde{\mathscr{P}}_{\mathrm{Fe}}^{L}\right]$ and the slope in equation $(24)$ is $\left(\tilde{\mathscr{P}}_{\mathrm{Fe}}^{\mathrm{VMS}}\right.$ $\left.+\tilde{\mathscr{P}}_{\mathrm{Fe}}^{\mathrm{L}}\right) / \tilde{\mathscr{P}}_{\mathrm{Fe}}^{\mathrm{L}}$. Table 2 shows that $\mathrm{SNe} \mathrm{II}(L)$ contributed $\sim 3 \%-10 \%$ of the $\mathrm{Fe}$ in stars with $[\mathrm{Fe} / \mathrm{H}]<-3$. Thus, $\left(\widetilde{\mathscr{P}}_{\mathrm{Fe}}^{\mathrm{VMS}}+\tilde{\mathscr{P}}_{\mathrm{Fe}}^{L}\right) / \tilde{\mathscr{P}}_{\mathrm{Fe}}^{L} \sim 10-30$. This results in a rather dramatic jump of $\sim 1-1.5$ dex between the evolutionary tracks of $\log \epsilon(\mathrm{Ba})$ at $[\mathrm{Fe} / \mathrm{H}]<-3$ and $[\mathrm{Fe} / \mathrm{H}]>-2.5$. By using the $\mathrm{Ba}$ and $\mathrm{Fe}$ yields of $\mathrm{SNe} \mathrm{II}(H)$ and $\mathrm{II}(L)$ in Table 1, the full trajectory of $\log \epsilon(\mathrm{Ba})$ is calculated from equation (20) (see $\S 4.2)$ and shown in Figure 1 for $\left(\widetilde{\mathscr{P}}_{\mathrm{Fe}}^{\mathrm{VMS}}+\tilde{\mathscr{P}}_{\mathrm{Fe}}^{L}\right) / \widetilde{\mathscr{P}}_{\mathrm{Fe}}^{L}=10$ (solid curve) and 20 (long-dashed curve). We recognize that the sparsity of data at $[\mathrm{Fe} / \mathrm{H}]<-3$ does not permit an accurate determination of the evolution in this region. We consider that this evolution may be represented by the corresponding parts of the trajectories shown in Figure 1 as the jump between the trends of the data at $[\mathrm{Fe} / \mathrm{H}]<-3$ and $[\mathrm{Fe} / \mathrm{H}]>-2.5$ is adequately reproduced.

\subsection{General Continuous Model}

The above continuous model can be extended to the general case including SN Ia contributions to $\mathrm{E}$ and $\mathrm{Fe}$ in a straightforward manner. For convenience of discussion, we designate the following regions for evolution of $E$ relative to $\mathrm{Fe}:(\mathrm{A})[\mathrm{Fe} / \mathrm{H}]<-3$ with contributions to $\mathrm{E}$ and $\mathrm{Fe}$ from VMSs and $\mathrm{SNe} \mathrm{II}(H)$ and $\mathrm{II}(L),(\mathrm{B})-3 \lesssim[\mathrm{Fe} / \mathrm{H}] \lesssim-2.5$ and $(\mathrm{C})-2.5<[\mathrm{Fe} / \mathrm{H}]<-1$ with contributions from $\mathrm{SNe}$ $\mathrm{II}(H)$ and $\mathrm{II}(L)$, and $(\mathrm{D})[\mathrm{Fe} / \mathrm{H}] \gtrsim-1$ with contributions from $\mathrm{SNe} \mathrm{II}(H), \mathrm{II}(L)$, and Ia. A typical evolutionary trajectory of $\log \epsilon(\mathrm{E})$ in these four regions is shown in Figure $5 a$ (solid curve). As discussed earlier, the trajectory in region A follows a straight line of unit slope determined by the ratio of the total rates per $\mathrm{H}$ atom for production of $\mathrm{E}$ and $\mathrm{Fe}$ by VMSs and $\mathrm{SNe} \mathrm{II}(H)$ and $\mathrm{II}(L)$. As a result of the termination of VMS activities at $[\mathrm{Fe} / \mathrm{H}] \approx-3$ and the corresponding change in the ratio of the total production rates per $\mathrm{H}$ atom for $\mathrm{E}$ and $\mathrm{Fe}$, the trajectory in region $\mathrm{B}$ shifts from the line in region A. Note that the termination of VMS activities also greatly decreases the dilution mass for the SN II ejecta to the standard value. Thus, the $\log \epsilon(\mathrm{E})$ and $[\mathrm{Fe} / \mathrm{H}]$ values produced by VMSs and $\mathrm{SNe} \operatorname{II}(H)$ and $\mathrm{II}(L)$ up to $[\mathrm{Fe} / \mathrm{H}] \approx-3$ are soon overwhelmed by the additional contributions from $\mathrm{SNe} \mathrm{II}(H)$ and $\mathrm{II}(L)$ in region $\mathrm{B}$. This typically occurs at $[\mathrm{Fe} / \mathrm{H}] \sim-2.5$ when a few $\mathrm{SNe} \mathrm{II}(L)$ have occurred. The trajectory in region $\mathrm{C}$ then follows the straight line of unit slope determined by the ratio of the total rates per $\mathrm{H}$ atom for production of $\mathrm{E}$ and $\mathrm{Fe}$ by $\mathrm{SNe}$ $\mathrm{II}(H)$ and $\mathrm{II}(L)$. At $[\mathrm{Fe} / \mathrm{H}] \sim-1$, SNe Ia began to occur with major $\mathrm{Fe}$ additions. Thus, the ratio of the total production rates per $\mathrm{H}$ atom for $\mathrm{E}$ and $\mathrm{Fe}$ may change again at the onset of SN Ia contributions. The trajectory in region D then may shift from the line in region $\mathrm{C}$ and reach another asymptotic straight line of unit slope after a transition zone.

In order to apply the general continuous model to the data on various elements, we need to know the ratios of the total production rates per $\mathrm{H}$ atom for $\mathrm{E}$ and $\mathrm{Fe}$ in regions $\mathrm{A}, \mathrm{B}$ (or C), and D:

$$
\gamma_{\mathrm{A}}(\mathrm{E}) \equiv \frac{\tilde{\mathscr{P}}_{\mathrm{E}}^{\mathrm{VMS}}+\tilde{\mathscr{P}}_{\mathrm{E}}^{H}+\tilde{\mathscr{P}}_{\mathrm{E}}^{L}}{\tilde{\mathscr{P}}_{\mathrm{Fe}}^{\mathrm{VMS}}+\tilde{\mathscr{P}}_{\mathrm{Fe}}^{L}}
$$

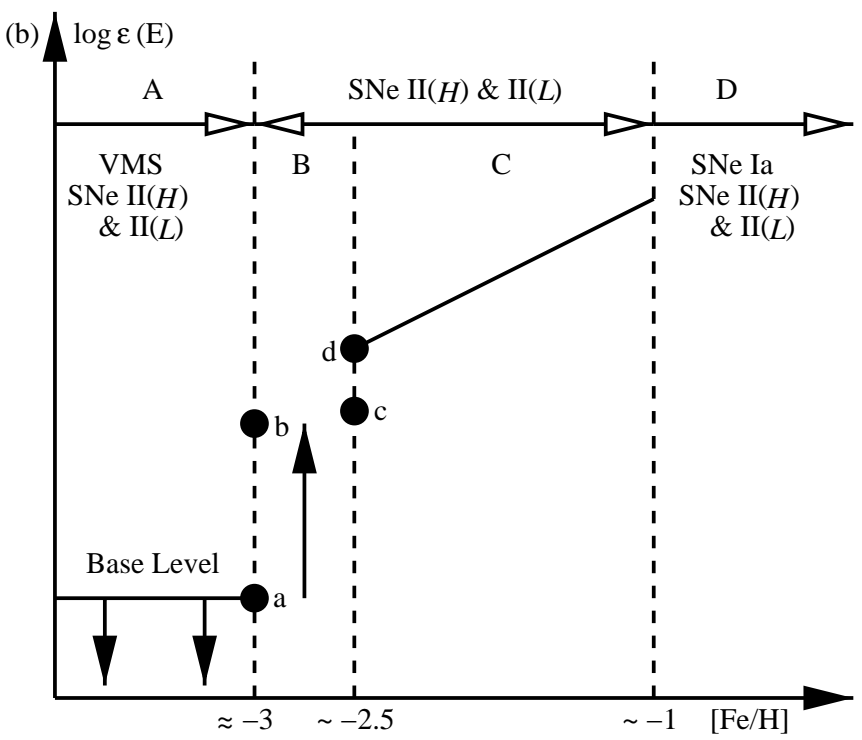

FIG. 5. - (a) Schematic illustration of continuous evolution of $\log \epsilon(\mathrm{E})$ as a function of $[\mathrm{Fe} / \mathrm{H}]$ in four different regions $(\mathrm{A}, \mathrm{B}, \mathrm{C}$, and D). The production sources in each region are indicated. The cusps at $[\mathrm{Fe} / \mathrm{H}] \approx-3$ and -1 are due to the transitions where production in one region changes to that in the next (see text). (b) Schematic illustration of the effects of discrete events on the evolution of $r$-process elements such as Ba. As a result of the enormous dilution mass associated with VMS activities in region A, the typical abundances in this region are well below the base level resulting from a single $\mathrm{SN}$ II $(H)$ with the standard dilution mass. The VMS activities cease at $[\mathrm{Fe} / \mathrm{H}] \approx-3$, and the dilution mass drops sharply to the standard value. Point (a) in region $B$ corresponds to a single $\mathrm{SN} \mathrm{II}(H)$ and point (b) to $10 \mathrm{SNe} \mathrm{II}(H)$. As $\mathrm{SNe} \mathrm{II}(H)$ produce no Fe, the occurrence of these events in region B follows the upward-pointing arrow from point (a) to point (b). Point (c) corresponds to a single SN II $(L)$ and point (d) to $10 \mathrm{SNe} \operatorname{II}(H)$ and a single SN $\operatorname{II}(L)$, all with the standard dilution mass. The continuous approximation fails and a discrete model must be used in region B (see text). 


$$
\begin{aligned}
\gamma_{\mathrm{BC}}(\mathrm{E}) & \equiv \frac{\mathscr{P}_{\mathrm{E}}^{H}+\mathscr{P}_{\mathrm{E}}^{L}}{\mathscr{P}_{\mathrm{Fe}}^{L}}=\frac{\tilde{\mathscr{P}}_{\mathrm{E}}^{H}+\tilde{\mathscr{P}}_{\mathrm{E}}^{L}}{\tilde{\mathscr{P}}_{\mathrm{Fe}}^{L}}, \\
\gamma_{\mathrm{D}}(\mathrm{E}) & \equiv \frac{\mathscr{P}_{\mathrm{E}}^{H}+\mathscr{P}_{\mathrm{E}}^{L}+\mathscr{P}_{\mathrm{E}}^{\mathrm{I}}}{\mathscr{P}_{\mathrm{Fe}}^{L}+\mathscr{P}_{\mathrm{Fe}}^{\mathrm{I}}},
\end{aligned}
$$

where $\mathscr{P}_{\mathrm{E}}^{\mathrm{I}}$ and $\mathscr{P}_{\mathrm{Fe}}^{\mathrm{I}}$ are the rates per $\mathrm{H}$ atom for production of $\mathrm{E}$ and $\mathrm{Fe}$, respectively, by SNe Ia. For regions $\mathrm{B}$ and $\mathrm{C}$ we may estimate $\gamma_{\mathrm{BC}}(\mathrm{E})$ directly from the $\mathrm{SN} \mathrm{II}(H)$ and $\mathrm{II}(L)$ yields in Table 1 as

$$
\gamma_{\mathrm{BC}}(\mathrm{E})=\frac{10 \times 10^{\log \epsilon_{H}(\mathrm{E})}+10^{\log \epsilon_{L}(\mathrm{E})}}{10^{\log \epsilon_{L}(\mathrm{Fe})}}
$$

where we have used the frequency ratio of $10: 1$ for $\mathrm{SNe} \mathrm{II}(H)$ and $\mathrm{II}(L)$ (e.g., QW01c). For region A the ratio $\gamma_{\mathbf{A}}(\mathrm{E})$ can be estimated from

$$
\frac{\gamma_{\mathrm{A}}(\mathrm{E})}{\gamma_{\mathrm{BC}}(\mathrm{E})}=\left(\frac{\widetilde{\mathscr{P}}_{\mathrm{E}}^{\mathrm{VMS}}+\tilde{\mathscr{P}}_{\mathrm{E}}^{H}+\tilde{\mathscr{P}}_{\mathrm{E}}^{L}}{\widetilde{\mathscr{P}}_{\mathrm{E}}^{H}+\widetilde{\mathscr{P}}_{\mathrm{E}}^{L}}\right)\left(\frac{\tilde{\mathscr{P}}_{\mathrm{Fe}}^{L}}{\widetilde{\mathscr{P}}_{\mathrm{Fe}}^{\mathrm{VMS}}+\widetilde{\mathscr{P}}_{\mathrm{Fe}}^{L}}\right)=\frac{f_{H L}(\mathrm{Fe})}{f_{H L}(\mathrm{E})}
$$

where $f_{H L}(\mathrm{E})$ and $f_{H L}(\mathrm{Fe})$ are the fractions of $\mathrm{E}$ and $\mathrm{Fe}$ at $[\mathrm{Fe} / \mathrm{H}]<-3$ contributed by $\mathrm{SNe} \mathrm{II}(H)$ and $\mathrm{II}(L)$ with typical values given in Table 2 .

Now to estimate $\gamma_{\mathrm{D}}(\mathrm{E})$ for region $\mathrm{D}$, we must consider the rates per $\mathrm{H}$ atom for Fe production by both $\mathrm{SNe} \mathrm{II}(L)$ and

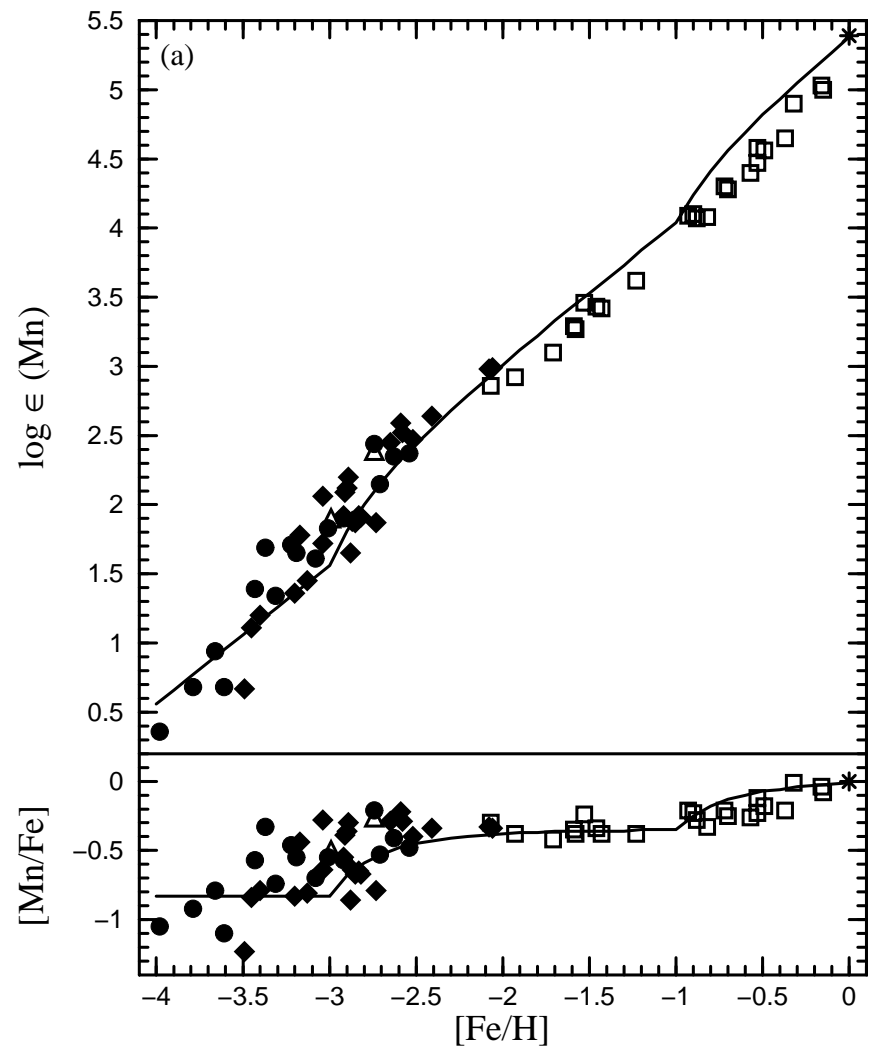

Ia, $\mathscr{P}_{\mathrm{Fe}}^{L}$ and $\mathscr{P}_{\mathrm{Fe}}^{\mathrm{I}}$. Once the ratio $\mathscr{P}_{\mathrm{Fe}}^{\mathrm{I}} / \mathscr{P}_{\mathrm{Fe}}^{L}$ is determined, $\gamma_{\mathrm{D}}(\mathrm{E})$ can be estimated from

$$
\gamma_{\mathrm{D}}(\mathrm{E})=\frac{\gamma_{\mathrm{BC}}(\mathrm{E})+\left(\mathscr{P}_{\mathrm{E}}^{\mathrm{I}} / \mathscr{P P}_{\mathrm{Fe}}^{\mathrm{I}}\right)\left(\mathscr{P}_{\mathrm{Fe}}^{\mathrm{I}} / \mathscr{P}_{\mathrm{Fe}}^{L}\right)}{1+\left(\mathscr{P}_{\mathrm{Fe}}^{\mathrm{I}} / \mathscr{P}_{\mathrm{Fe}}^{L}\right)}
$$

by using the SN Ia yield pattern in Table 1 . We recognize that the progenitors of SNe $\mathrm{II}(L)$ and Ia evolve very differently and the ratio $\mathscr{P}_{\mathrm{Fe}}^{\mathrm{I}} / \mathscr{P}_{\mathrm{Fe}}^{L}$ may not be constant over Galactic history. For simplicity, we assume a constant $\mathscr{P}_{\mathrm{Fe}}^{\mathrm{I}} / \mathscr{P}_{\mathrm{Fe}}^{L}$ and estimate its value by making use of the timescales associated with $\mathrm{SN} \mathrm{II}(L)$ and Ia activities. We have assumed that $\mathrm{SNe} \operatorname{II}(L)$ are responsible for $\approx \frac{1}{3}$ of the solar $\mathrm{Fe}$ inventory and SNe Ia for the remaining fraction of $\approx \frac{2}{3}$. Therefore, we have $\mathscr{P}_{\mathrm{Fe}}^{\mathrm{I}} T_{\mathrm{I}} /\left(\mathscr{P}_{\mathrm{Fe}}^{L} T_{\mathrm{II}}\right) \approx 2$, where $T_{\mathrm{I}}$ and $T_{\mathrm{II}}$ are the periods of SN Ia and II $(L)$ production prior to SSF. The ratio $T_{\mathrm{I}} / T_{\mathrm{II}}$ is less than unity and depends on the time required for a sufficient number of low-mass stars to evolve in binaries to provide SN Ia precursors. This time may be $\sim 1-3 \times 10^{9} \mathrm{yr}$, to be compared with $T_{\text {II }} \sim 10^{10} \mathrm{yr}$. We consider that $0.7 \lesssim T_{\mathrm{I}} / T_{\mathrm{II}} \lesssim 1$ and use $\mathscr{P}_{\mathrm{Fe}}^{\mathrm{I}} / \mathscr{P}_{\mathrm{Fe}}^{L}=2.5$ in equation (28) to estimate $\gamma_{\mathrm{D}}(\mathrm{E})$.

Examples of the full evolutionary trajectories for the general continuous model over the range of $-4 \leq$ $[\mathrm{Fe} / \mathrm{H}] \leq 0$ are shown in Figure 6 for $\mathrm{Mn}$ and Co along with the available data on these two elements. For comparison we also show the same results in the conventional $[\mathrm{E} / \mathrm{Fe}]$ representation. The cusps in the curves at $[\mathrm{Fe} / \mathrm{H}]=-3$ and -1 are due to the transitions where production in one regime changes to that in the next as described above. The intrinsic yield patterns of the different

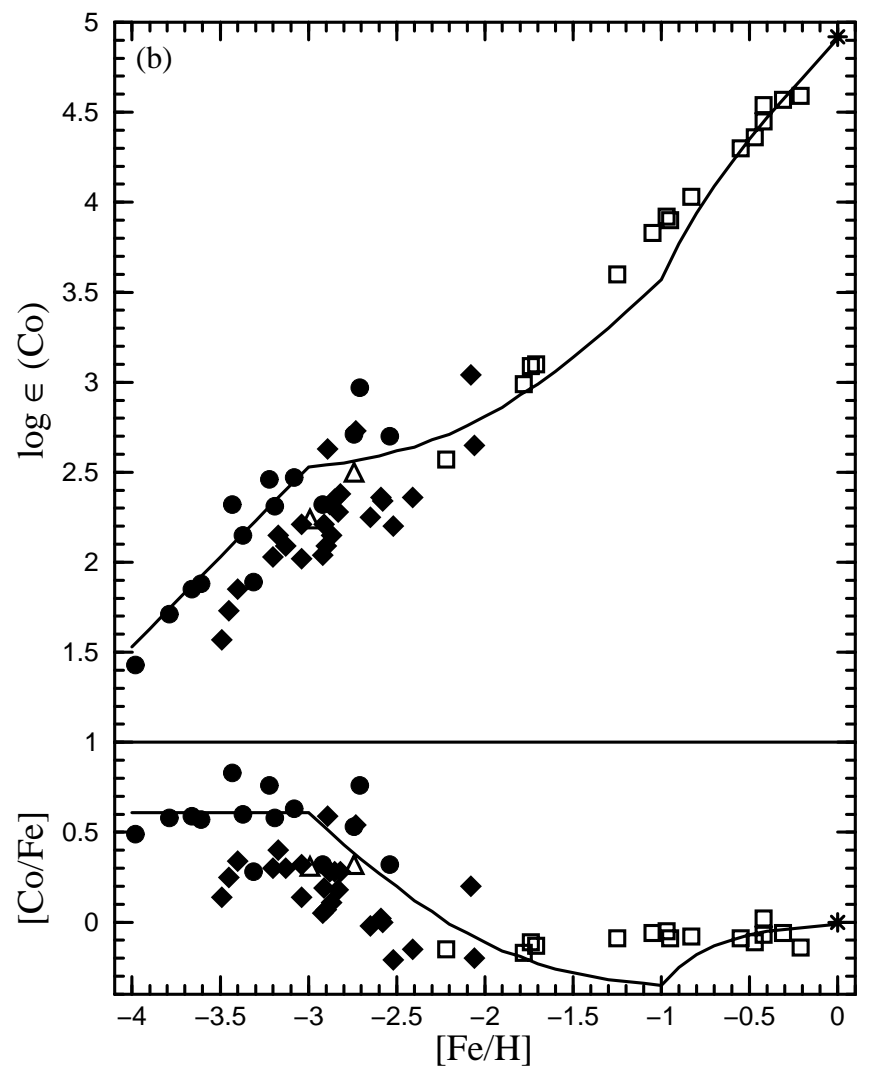

Fig. 6.-(a) Evolution of Mn relative to Fe for the continuous model (solid curves) compared with the data (filled circles: Ryan et al. 1996; Norris et al. 2001; filled diamonds: McWilliam et al. 1995; open squares: Gratton 1989; open triangles: Westin et al. 2000). The asterisk corresponds to the solar data. The parameters used are $f_{H L}(\mathrm{Mn})=0.3, f_{H L}(\mathrm{Fe})=0.1$, and $\mathscr{P}_{\mathrm{Fe}}^{\mathrm{I}} / \mathscr{P}_{\mathrm{Fe}}^{L}=2.5$. No attempt is made to fit the model to the data. (b) Same as $(a)$, but for Co [with open squares indicating data from Gratton \& Sneden 1991 and with only one different parameter $f_{H L}(\mathrm{Co})=0.01$ for the model]. 
sources are unchanged. Note that the yield pattern of $\mathrm{Mn}$, $\mathrm{Fe}$, and Co for VMSs is distinct from that for $\mathrm{SNe} \mathrm{II}(L)$. As VMSs turn off and SNe II continue with increased production rates per $\mathrm{H}$ atom at $[\mathrm{Fe} / \mathrm{H}]=-3$, the intrinsic differences in the yield patterns of these two sources result in different changes in the ratios of the total production rates per $\mathrm{H}$ atom and hence in opposite trends for the evolution of $\mathrm{Mn}$ and $\mathrm{Co}$ in the region of $-4<[\mathrm{Fe} / \mathrm{H}]<-2.5$. These opposite trends as seen in the observational data have drawn the attention of Nakamura et al. (1999). They sought to explain the observations by changing the yields of the Fe group elements through different mass cuts in $a b$ initio models of SN II nucleosynthesis. In our approach, these trends are simply explained by the existence of VMSs at $-4 \lesssim[\mathrm{Fe} / \mathrm{H}]<-3$ with a yield pattern of $\mathrm{Mn}, \mathrm{Fe}$, and Co distinct from that of $\mathrm{SNe} \mathrm{II}(L)$. The case of $\mathrm{Cr}$ is similar to $\mathrm{Mn}$ and can be explained in the same manner by our approach.

The general continuous model can be used to discuss all the other elements in the group from $\mathrm{Na}$ to $\mathrm{Ni}$ as in the above examples for Mn and Co. Summary of the relevant data can be found in the figures of McWilliam et al. (1995) and Norris et al. (2001). A common feature for all these elements is that the abundance ratios of other elements to $\mathrm{Fe}$ become rather well defined when $[\mathrm{Fe} / \mathrm{H}] \sim-2.5$ is reached.

\subsection{Effects of Discrete Events}

If there is more than one source for $\mathrm{E}$ and $\mathrm{Fe}$ as in the case of $\mathrm{Ba}$, the continuous approximation is good when a sufficient number of production events of each kind have occurred in the ISM. If this requirement is not satisfied, a discrete model should be used. The Ba data in Figure 1 show that $\log \epsilon(\mathrm{Ba})$ increases from approximately -2.8 at $[\mathrm{Fe} / \mathrm{H}] \sim-4$ to approximately -1.8 at $[\mathrm{Fe} / \mathrm{H}] \sim-3$. Thus, the number of Ba-producing $\mathrm{SNe} \operatorname{II}(H)$ and $\mathrm{II}(L)$ increased by a factor of $\approx 10$ over the range of $-4 \lesssim[\mathrm{Fe} / \mathrm{H}]<-3$, and the continuous approximation should become good as $[\mathrm{Fe} / \mathrm{H}]$ approaches -3 . At $[\mathrm{Fe} / \mathrm{H}] \gtrsim-3$, the dilution mass for the SN II ejecta decreased greatly to the standard value and a single SN $\mathrm{II}(L)$ would result in $[\mathrm{Fe} / \mathrm{H}] \approx-2.5$. Thus, we expect that the continuous approximation should also hold at $[\mathrm{Fe} / \mathrm{H}]>-2.5$ after a few $\mathrm{SNe} \mathrm{II}(L)$ had occurred with the standard dilution mass. The evolutionary trends exhibited by the $\mathrm{Ba}$ data at $[\mathrm{Fe} / \mathrm{H}]<-3$ and $[\mathrm{Fe} / \mathrm{H}]>-2.5$ are adequately described by two straight lines of unit slope offset by $\sim 1-1.5$ dex as calculated from the continuous model (see Fig. 1).

The effects of discrete events are most pronounced in region $\mathrm{B}$ of $-3 \lesssim[\mathrm{Fe} / \mathrm{H}] \lesssim-2.5$ for $r$-process elements such as $\mathrm{Ba}$ as illustrated schematically in Figure $5 b$. As a result of the enormous dilution mass for the SN II ejecta in region $\mathrm{A}$, the typical $\log \epsilon(\mathrm{E})$ values for $r$-process elements in this region are significantly below the "base level" resulting from a single SN $\mathrm{II}(H)$ with the standard dilution mass. This is indicated by the downward-pointing arrows from the base level in Figure $5 b$. After the cessation of VMS activities at $[\mathrm{Fe} / \mathrm{H}] \approx-3$, the standard dilution mass applies. A single $\mathrm{SN} \mathrm{II}(H)$ would then correspond to point (a) and $10 \mathrm{SNe} \mathrm{II}(H)$ to point $(\mathrm{b})$ in region $\mathrm{B}$. As $\mathrm{SNe} \mathrm{II}(H)$ produce no $\mathrm{Fe}$, the occurrence of these events in region $\mathrm{B}$ is represented by the upward-pointing arrow from point (a) to point (b). A single $\mathrm{SN} \mathrm{II}(L)$ with the standard dilution mass would correspond to point (c) at the boundary between regions $\mathrm{B}$ and $\mathrm{C}$ because it produces both $\mathrm{Fe}$ and, e.g., $\mathrm{Ba}$. As $\mathrm{SNe} \mathrm{II}(H)$ are 10 times more frequent than $\mathrm{SNe} \mathrm{II}(L)$, point (d) corresponding to $10 \mathrm{SNe} \mathrm{II}(H)$ and a single $\mathrm{SN}$ $\mathrm{II}(L)$ would lie on the trend line in region $\mathrm{C}$ as calculated from the continuous model. For an $\mathrm{SN} \operatorname{II}(L)$ frequency of $\left(10^{8} \mathrm{yr}\right)^{-1}$ in a standard dilution mass of ISM, region B corresponds to a period of $\lesssim 10^{8}$ yr during which SNe II $(H)$ but not $\mathrm{SNe} \mathrm{II}(L)$ would occur with a high probability (WQ00a). Thus, the continuous approximation does not apply to region $\mathrm{B}$ and the trajectory in this region calculated from the continuous model does not provide a physical description of the data on, e.g., Ba. The large scatter in, e.g., $\log \epsilon(\mathrm{Ba})$ over the narrow range of $-3 \lesssim[\mathrm{Fe} / \mathrm{H}] \lesssim-2.5$ is accounted for by the occurrence of $\sim 1-10 \mathrm{SNe} \mathrm{II}(H)$ that produce $\mathrm{Ba}$ but no $\mathrm{Fe}$ in the discrete model (WQ00a).

\section{DISCUSSION AND CONCLUSIONS}

Based on a phenomenological model and the available data on abundances in metal-poor stars, we have determined the yields of the elements from $\mathrm{Na}$ to $\mathrm{Ni}$ for $\mathrm{SNe}$ $\mathrm{II}(H)$ and $\mathrm{II}(L)$ and the yield patterns of the same elements for SNe Ia and VMSs. In a previous companion paper (QW01c) we determined the yields of $\mathrm{SNe} \operatorname{II}(H)$ and $\mathrm{II}(L)$ and the composition of the $P$ inventory for $r$-process elements ( $\mathrm{Sr}$ and above). The $P$ inventory of $r$-process elements is now considered to be the result of concomitant production by $\mathrm{SNe} \mathrm{II}(H)$ and $\mathrm{II}(L)$ when VMSs were active. The $P$ inventory of non-neutron capture elements (possibly including $\mathrm{Sr}$ ) is considered to be the result of major production by VMSs with small contributions from $\mathrm{SNe}$ II $(H)$ and $\operatorname{II}(L)$. The results presented here and in QW01c show that the abundances of a large number of elements (of both rapid neutron capture and non-neutron capture origins) in any metal-poor star with $-3 \lesssim[\mathrm{Fe} / \mathrm{H}]<-1$ can be almost quantitatively determined from the observed $[\mathrm{Fe} / \mathrm{H}]$ and $\log \epsilon(\mathrm{Eu})$ values for the star by using the threecomponent model including the $P$ inventory and the contributions from $\mathrm{SNe} \operatorname{II}(H)$ and $\mathrm{II}(L)$. A similar approach also applies to stars with $-4 \lesssim[\mathrm{Fe} / \mathrm{H}]<-3$, for which the contributions from VMSs are explicitly included and the contributions from $\mathrm{SNe} \mathrm{II}(H)$ and $\mathrm{II}(L)$ are determined by using the observed $\log \epsilon(\mathrm{Ba})$ value as the index of $r$-process production.

By using the three-component model, it is shown that $\mathrm{SNe} \mathrm{II}(H)$ control the abundances of all the heavy $r$-process elements above $\mathrm{Ba}$ but contribute almost nothing to the inventory of the elements between $\mathrm{C}$ and Ge. The solar inventory of all the elements from $\mathrm{O}$ to $\mathrm{V}$ and the solar $r$-process inventory of all the elements from $\mathrm{Sr}$ to $\mathrm{Ba}$ are almost quantitatively accounted for by $\mathrm{SNe} \mathrm{II}(L)$. The majority of the solar inventory of the $\mathrm{Fe}$ group elements ( $\mathrm{Cr}, \mathrm{Mn}, \mathrm{Fe}, \mathrm{Co}$, and $\mathrm{Ni}$ ) is contributed by $\mathrm{SNe} \mathrm{Ia}$. The metal inventory of the universe at $[\mathrm{Fe} / \mathrm{H}]<-3$ was dominated by the contributions from VMSs. Compared with SNe II $(L)$, VMSs appear to have an almost identical yield pattern but with a distinct deficiency of $\mathrm{Na}, \mathrm{Al}, \mathrm{V}, \mathrm{Cr}$, and $\mathrm{Mn}$ and a distinct overproduction of Co. All of these discrepant elements except for $\mathrm{Cr}$ are of odd atomic number. It is found that the metallicity of $[\mathrm{Fe} / \mathrm{H}] \approx-3$ represents the state when the occurrence of VMSs was essentially terminated. While some formation of normal stars including SN II progenitors and low-mass stars occurred prior to the 
achievement of $[\mathrm{Fe} / \mathrm{H}] \approx-3$, the onset of major formation of normal stars appears to be at this metallicity. The rapid rise in the $\mathrm{Ba}$ abundance at $[\mathrm{Fe} / \mathrm{H}] \approx-3$ (see Fig. 1) is due to the cessation of VMS activities and the onset of more rapid normal star formation. The mass of ISM with which the nucleosynthetic products of VMSs and SNe II are mixed in the domain of $[\mathrm{Fe} / \mathrm{H}]<-3$ is much larger (by a factor of $\sim 25-300$ ) than that for SNe II in the domain of $[\mathrm{Fe} / \mathrm{H}] \gtrsim-3$ where VMSs ceased playing any significant role.

The above conclusions have implications in four distinct areas. The yields derived here should serve as (1) templates with which the results of ab initio nucleosynthetic models of SNe II and VMSs may be compared, (2) predictions for the abundances relative to $\mathrm{Fe}$ in stars with ultralow metallicities $([\mathrm{Fe} / \mathrm{H}]<-3)$, (3) input for discussing the cosmological aspects of condensation and dispersion of baryonic matter that led to the formation of galaxies and other aggregations of baryonic matter in the universe, and (4) basis for considering the general chemical evolution of the universe. In the following we will present a short summary on each of these issues based on the above findings.

\subsection{Implications for Nucleosynthetic Models of SNe II and VMSs}

\subsection{1. $\mathrm{SNe} I I(H)$}

It is commonly thought that in SNe II, the elements below $\mathrm{Si}$ are produced by hydrostatic burning during pre-SN evolution and those above (including $\mathrm{Si}$ ) are mostly produced by explosive burning associated with the shock propagation. In order not to produce a significant amount of the elements from $\mathrm{Na}$ to $\mathrm{Ni}$, an $\mathrm{SN} \mathrm{III}(H)$ must either have a pre-SN structure lacking substantial hydrostatic burning shells or, for some reason, have all the material below the He-burning shell fall back onto the central remnant. Stars with masses of $\sim 8-11 M_{\odot}$ have very thin shells at the end of their lives and explode as a result of the collapse of an $\mathrm{O}-\mathrm{Mg}-\mathrm{Ne}$ core instead of an Fe core (e.g., Nomoto 1984; Hillebrandt, Nomoto, \& Wolff 1984; Mayle \& Wilson 1988). These stars could be the progenitors for $\mathrm{SNe} \mathrm{II}(H)$. Mayle \& Wilson (1988) calculated that an SN from O-Mg$\mathrm{Ne}$ core collapse would only eject $\sim 0.042 M_{\odot}$ of matter and produce $\sim 0.002 M_{\odot}$ of Fe. By assuming that $10^{2} \mathrm{SNe}$ $\mathrm{II}(L)$ must enrich a standard dilution mass of $\approx 3 \times 10^{4} M_{\odot}$ with $\approx \frac{1}{3}$ of the solar $\mathrm{Fe}$ mass fraction of $\approx 10^{-3}$, the $\mathrm{Fe}$ yield of an $\mathrm{SN} \mathrm{II}(L)$ is estimated to be $\approx 0.1 M_{\odot}$. This is $\sim 50$ times larger than the $\mathrm{Fe}$ yield of an $\mathrm{SN}$ from $\mathrm{O}-\mathrm{Mg}-\mathrm{Ne}$ core collapse. Given the extremely low total amount of ejecta, it is reasonable to expect that $\mathrm{SNe}$ from $\mathrm{O}-\mathrm{Mg}-\mathrm{Ne}$ core collapse also do not produce any significant amount of other elements below the $\mathrm{Fe}$ group. On the other hand, we note that neutrino emission from the neutron star produced in any core collapse would drive a small amount of material from the neutron star crust. This neutrino-driven wind has been suggested as a site of the $r$-process (e.g., Woosley et al. 1994) although it remains to be seen if adequate conditions for the $r$-process can be obtained in the wind (e.g., Qian \& Woosley 1996; Hoffman, Woosley, \& Qian 1997). We consider that $\mathrm{SNe}$ from $\mathrm{O}-\mathrm{Mg}$-Ne core collapse may correspond to $\mathrm{SNe} \mathrm{II}(H)$ or at least a subset (see also Wheeler, Cowan, \& Hillebrandt 1998). Further studies of these objects are extremely important as most of the research on SNe II tends to focus on progenitors with masses of 12-40 $M_{\odot}$ that lead to explosion from $\mathrm{Fe}$ core collapse.
Another possibility to accommodate the nucleosynthetic requirements of $\mathrm{SNe} \mathrm{II}(H)$ is that $\mathrm{SNe}$ II from $\mathrm{Fe}$ core collapse would suffer severe fallback of processed material as emphasized by Woosley \& Weaver (1995). It has been argued that SN 1997D, which ejected only $0.002 M_{\odot}$ of Fe, was such a case (Benetti et al. 2001). The problem with such a scenario for $\mathrm{SNe} \mathrm{II}(H)$ is that the severe fallback would be an obstacle for ejection of the $r$-process material from the innermost regions of the SN. In any case, we consider that many issues remain to be explored concerning the fallback and possible production and ejection of $r$-process material in $\mathrm{SNe}$ from $\mathrm{Fe}$ core collapse.

Given the validity of our model, we require that $\mathrm{SNe} \mathrm{II}(H)$ be the dominant source of the heavy $r$-process elements above $\mathrm{Ba}$ but contribute very little of the other elements, especially those between $\mathrm{C}$ and $\mathrm{Ge}$. We also require $\mathrm{SNe} \mathrm{II}(H)$ to be the frequent kind of SNe II. Regardless of the theoretical issues involved in addressing the existence of such objects, clear predictions can be made for the observational consequences of $\mathrm{SNe} \mathrm{II}(H)$. First of all, $\mathrm{Fe}$ is mostly produced as the radioactive ${ }^{56} \mathrm{Ni}$, whose decay powers the light curve of an SN II at late stages. The lack of Fe production by $\mathrm{SNe} \mathrm{II}(H)$ means that the light curves of such events have a very faint tail as in the case of SN 1997D. Based on the frequent occurrences of $\mathrm{SNe} \mathrm{II}(H)$ in our model, it is likely that future systematic long-term observations of SN II light curves may find a large number of cases like SN 1997D. Another observational signature of $\mathrm{SNe} \mathrm{II}(H)$ relies on the occurrences of these events in binaries. A low-mass companion would experience surface contamination when an $\mathrm{SN} \mathrm{II}(H)$ explodes in a binary. As $\mathrm{SNe} \mathrm{II}(H)$ produce mainly the heavy $r$-process elements above Ba but little else, the surface of the low-mass companion should be highly enriched in the heavy $r$-process elements but appear more or less normal in, e.g., $\mathrm{Na}$ to $\mathrm{Ni}$, when compared with other stars with similar $[\mathrm{Fe} / \mathrm{H}]$. This exactly fits the observations of CS 22892-052 as discussed in $\S 2$. We have also argued that CS 31082-001 is another example of surface contamination by the SN II $(H)$ ejecta in a binary (Qian \& Wasserburg 2001b) based on the highly enriched abundances of heavy $r$-process elements but very low value of $[\mathrm{Fe} / \mathrm{H}]=-2.9$ for this star (Cayrel et al. 2001). There are no available data on the elements in the group of $\mathrm{Na}$ to $\mathrm{Ni}$ other than $\mathrm{Fe}$ for CS 31082-001. As the value of $[\mathrm{Fe} / \mathrm{H}]=-2.9$ is almost the same as that for the $P$ inventory, we predict that future observations of this star should detect abundances similar to the $P$ inventory for all the other elements in the group of $\mathrm{Na}$ to Ni. The abundances of heavy $r$-process elements observed in CS 31082001 are extremely high and correspond to $n_{H} \sim 100$. Thus, the observations of $\mathrm{Na}$ to $\mathrm{Ni}$ in this star may be used to set a severe limit on the production of these elements by SNe II $(H)$. We urge that such observations be carried out soon.

\subsection{2. $S N e I I(L)$}

The SN II $(L)$ yields presented here are inferred from the data on a star with $[\mathrm{Fe} / \mathrm{H}]=-2.04$ corresponding to several contributing $\mathrm{SNe} \mathrm{II}(L)$. These yields almost exactly follow the solar abundance pattern up through V. This suggests that the SN $\mathrm{II}(L)$ yields may be interpreted as the contributions from a single type of event and that the yield pattern is constant, i.e., independent of metallicity over Galactic history. The extremely regular SN II $(L)$ yields pre- 

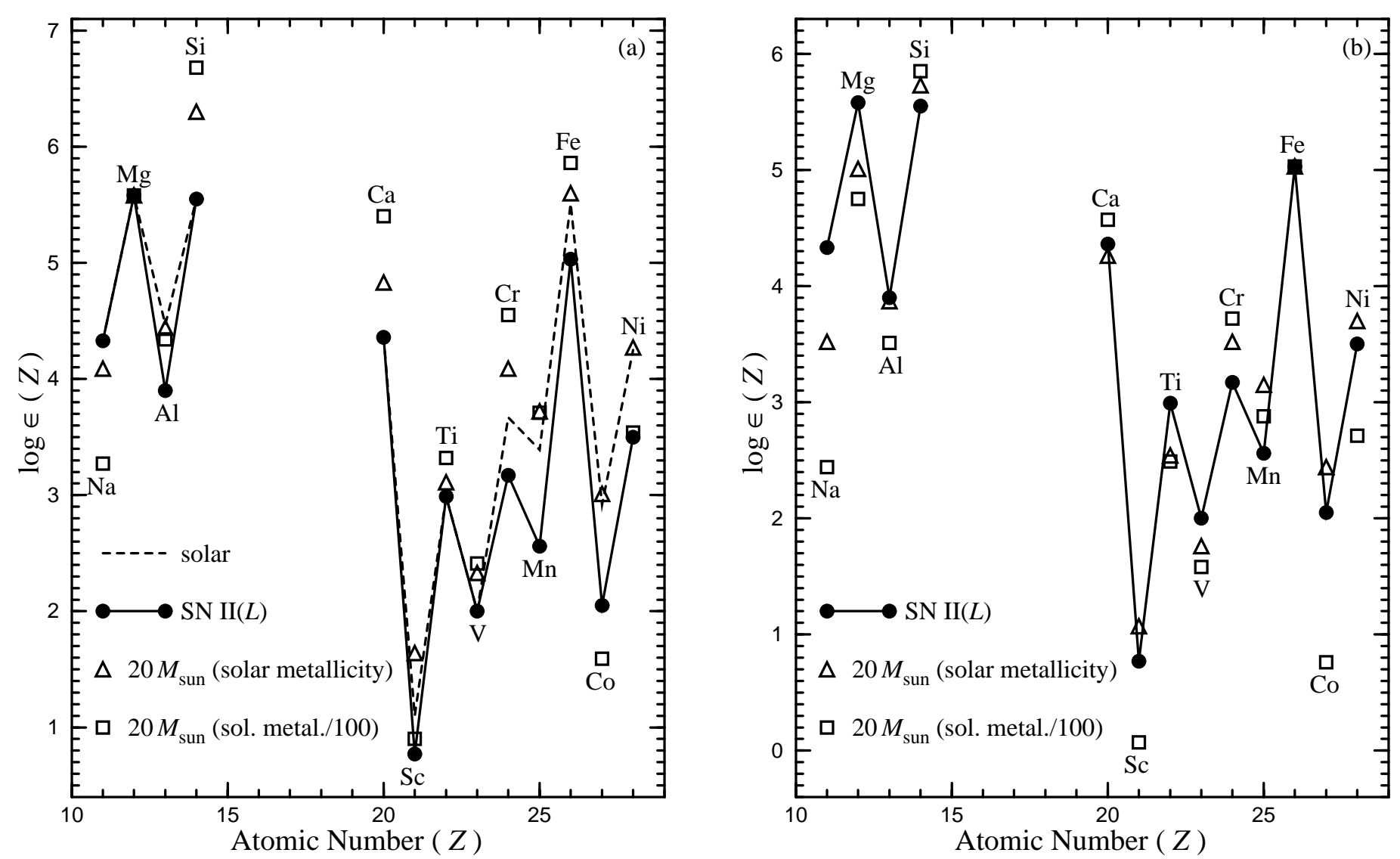

FIG. 7.- $\mathrm{SN} \mathrm{II}(L)$ yield pattern ( filled circles connected by solid curves) compared with the yield patterns calculated from models (Woosley \& Weaver 1995) of SNe II with $20 M_{\odot}$ progenitors of solar (triangles) and 0.01 times solar (squares) metallicities. The model yields have been shifted to pass through $\log \epsilon(\mathrm{Mg})=\log \epsilon_{L}(\mathrm{Mg})=5.58$ in panel $(a)$ and $\log \epsilon(\mathrm{Fe})=\log \epsilon_{L}(\mathrm{Fe})=5.03$ in panel $(b)$. The solar abundance pattern translated to pass through $\log \epsilon(\mathrm{Mg})=5.58$ (dashed curve) is also shown in panel $(a)$.

sented here may be compared with the yields calculated from nucleosynthetic models of SNe II, for which diversity appears to be the rule. Figure 7 compares the SN $\mathrm{II}(L)$ yields ( filled circles connected by solid curves) with the yield patterns calculated from models (Woosley \& Weaver 1995) of SNe II with $20 M_{\odot}$ progenitors of solar (triangles) and 0.01 times solar (squares) metallicities. The model yields have been shifted to pass through the $\mathrm{SN}$ II $(L)$ yield of $\mathrm{Mg}$ in Figure $7 a$ and that of $\mathrm{Fe}$ in Figure $7 b$. These two choices of the reference point are made to reflect that the elements below and above $\mathrm{Si}$ have different nucleosynthetic origins in $\mathrm{SNe}$ II. It can be seen that the model yields are dependent on metallicity and that both model yield patterns only resemble the $\mathrm{SN} \mathrm{II}(L)$ yield pattern in local regions.

To emphasize the regularity of $\mathrm{SN} \operatorname{II}(L)$ yields inferred from observations, we show in Figure 8 the data on $\log (\mathrm{Si} / \mathrm{Mg})$ over the wide range of $-4 \lesssim[\mathrm{Fe} / \mathrm{H}] \lesssim-1.3$. It can be seen that most $(\approx 75 \%)$ of the data are within a factor of 2 of $\mathrm{Si} / \mathrm{Mg}=1 \approx(\mathrm{Si} / \mathrm{Mg})_{\odot}$ and that there is no evidence for high $\mathrm{Si} / \mathrm{Mg}$ ratios of greater than 3 . In contrast to this regularity, the values of $\mathrm{Si} / \mathrm{Mg}$ in most models (e.g., Woosley \& Weaver 1995) vary over a wide range of $\sim 0.1-$ 10 . This variability is expected as $\mathrm{Mg}$ is produced by hydrostatic burning but $\mathrm{Si}$ by a mixture of hydrostatic and explosive burning. We emphasize that averaging over many $\mathrm{SNe}$ II does not provide a viable way to reconcile the diverse $\mathrm{Si} / \mathrm{Mg}$ ratios in the models with the uniform $\mathrm{Si} / \mathrm{Mg}$ values exhibited by the data. This is because the same uniformity of $\mathrm{Si} / \mathrm{Mg}$ applies to the data at $[\mathrm{Fe} / \mathrm{H}] \sim$
-2.5 , which corresponds to $\mathrm{Fe}$ contributions from a few $\mathrm{SNe} \mathrm{II}(L)$.

The modeling of explosive burning is extremely sensitive to many uncertainties in our understanding of $\mathrm{SNe}$ II as

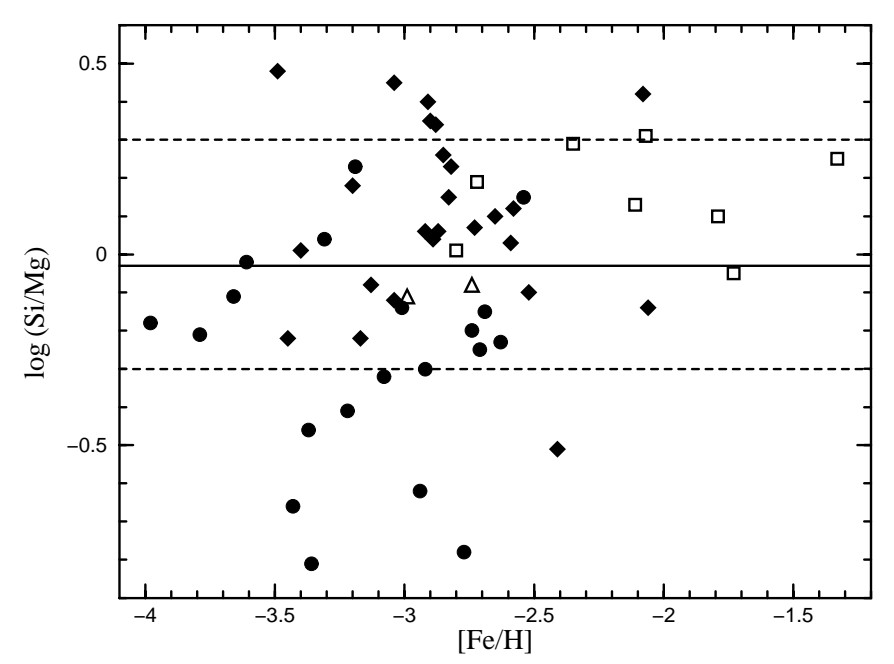

FIG. 8.-Observed $\log (\mathrm{Si} / \mathrm{Mg})$ values over the range of $-4 \lesssim[\mathrm{Fe} / \mathrm{H}] \lesssim-1.3$ ( filled circles: Ryan et al. 1996; Norris et al. 2001; filled diamonds: McWilliam et al. 1995; open squares: Gratton \& Sneden 1988; open triangles: Westin et al. 2000). The solid line represents the solar value of $\log (\mathrm{Si} / \mathrm{Mg})_{\odot}=-0.03$. Note that almost all $(\approx 75 \%)$ of the data lie within \pm 0.3 dex (between the dashed lines) of $\log (\mathrm{Si} / \mathrm{Mg})=0$. There is no evidence of very high $\mathrm{Si} / \mathrm{Mg}$ ratios. 
emphasized by Woosley \& Weaver (1995) and Thielemann, Nomoto, \& Hashimoto (1996). This sensitivity is commonly explored by adopting various mass cuts for the SN II ejecta. However, introduction of mass cuts deviates from the ab initio approach and must rely on empirical guidance such as the Fe yields inferred from SN II light curves. Note that the typical inferred $\mathrm{Fe}$ yields are $\sim 0.1 M_{\odot}$ (e.g., Thielemann et al. 1996), which are consistent with the Fe yield estimated for $\mathrm{SNe} \mathrm{II}(L)$ in the preceding discussion on $\mathrm{SNe} \mathrm{II}(H)$. It is conceivable that by choosing a mass cut to give the empirical Fe yield, SN II models would give more convergent yield patterns similar to the $\mathrm{SN} \mathrm{II}(L)$ yield pattern derived here from the observed abundances in metal-poor stars. A deeper issue then arises concerning the physics and astrophysics underlying the mass cut.

\subsubsection{VMSs}

We have shown that the yield pattern of VMSs is also quite regular. In particular, this pattern is almost identical to the $\mathrm{SN} \mathrm{II}(L)$ yield pattern except for the distinct deficiency at $\mathrm{Na}, \mathrm{Al}, \mathrm{V}, \mathrm{Cr}$, and $\mathrm{Mn}$, the overproduction of $\mathrm{Co}$, and the variable production of $\mathrm{Sr}$ (see Fig. 4). We will not discuss $\mathrm{Sr}$ any further. All the other discrepant elements are of odd atomic numbers except for $\mathrm{Cr}$. The underproduction of the elements of odd atomic numbers by VMSs is expected from the requirement of neutron excess to produce these elements. During the evolution of an SN II progenitor, significant weak interaction such as $\beta^{+}$decay and electron capture would take place during hydrostatic burning to increase the neutron excess. However, VMSs encounter pair instability after core He burning and there is no stage of

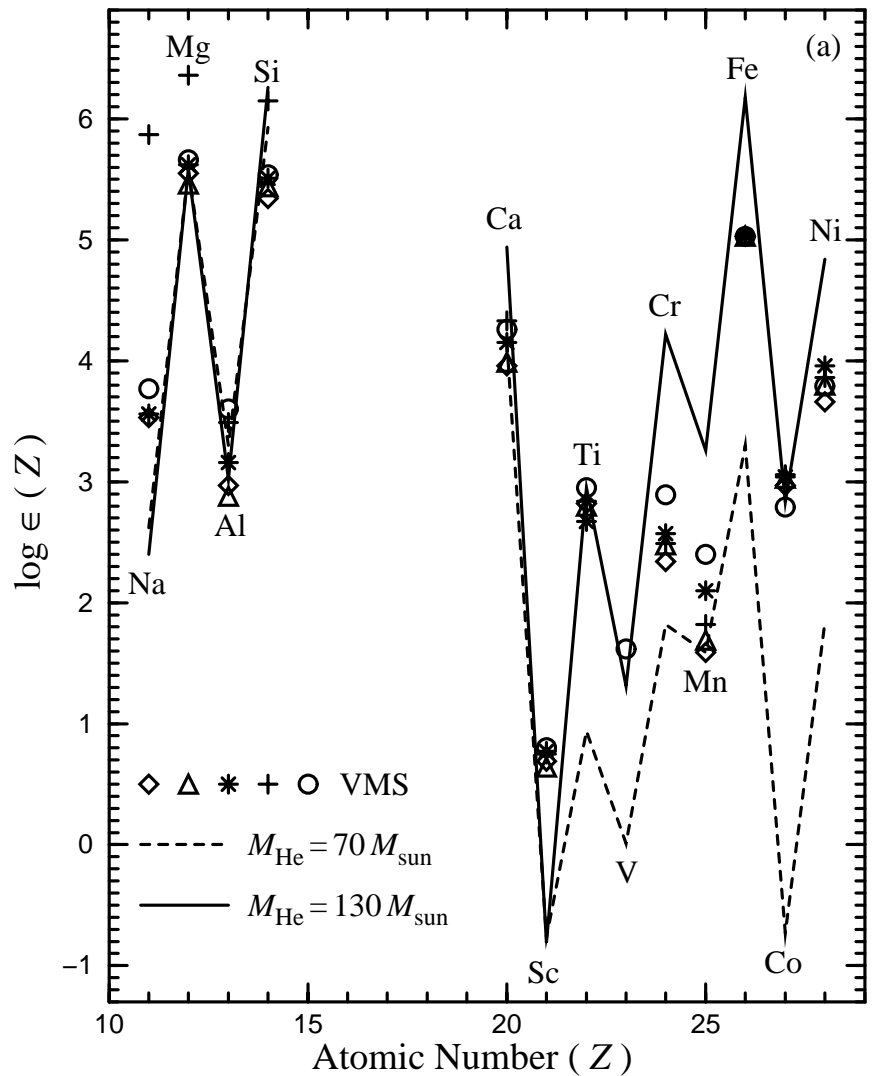

stable post-He burning to increase the neutron excess (Heger \& Woosley 2002). Thus, VMSs are expected to greatly underproduce the elements of odd atomic numbers relative to $\mathrm{SNe}$ II. However, the underproduction of $\mathrm{Cr}$ of even atomic number, the overproduction of $\mathrm{Co}$ of odd atomic number, and the similar production of $\mathrm{Sc}$ of odd atomic number by VMSs relative to $\mathrm{SNe} \operatorname{II}(L)$ as found here are not readily explained.

Figure 9 compares the VMS yield patterns inferred from the data on four stars with $-4 \lesssim[\mathrm{Fe} / \mathrm{H}]<-3$ and that derived from the $P$ inventory with the yields calculated from two limiting models (Heger \& Woosley 2002) of VMSs with $\mathrm{He}$ core masses of 70 (dashed curve) and $130 M_{\odot}$ (solid curve). The model yields have been shifted to pass through $\log \epsilon(\mathrm{Mg})=5.58 \quad$ [the location of most $\log \epsilon_{\mathrm{VMS}}(\mathrm{Mg})$ values] in Figure $9 a$ and $\log \epsilon(\mathrm{Fe})=5.03$ [the location of all $\log \epsilon_{\mathrm{VMS}}(\mathrm{Fe})$ values] in Figure $9 b$. These two choices of the reference point are made to compare the model yields with the yield patterns derived here in two different regions. It can be seen that below $\mathrm{Ti}$, the model yields are quite robust and are a fair representation of the yield pattern for elements of even atomic numbers as derived here. By contrast, the production of the elements above Ti in the models increases with the He core mass. It is also evident that the production of the elements of odd atomic numbers and $\mathrm{Cr}$ as derived here cannot be simply accounted for by the models in a quantitative manner. Comparison with models for intermediate $\mathrm{He}$ core masses does not change the basic conclusions.

The explosion energy in the VMS models of Heger \& Woosley (2002) lies in the range of $\sim 10^{52}-10^{53}$ ergs and

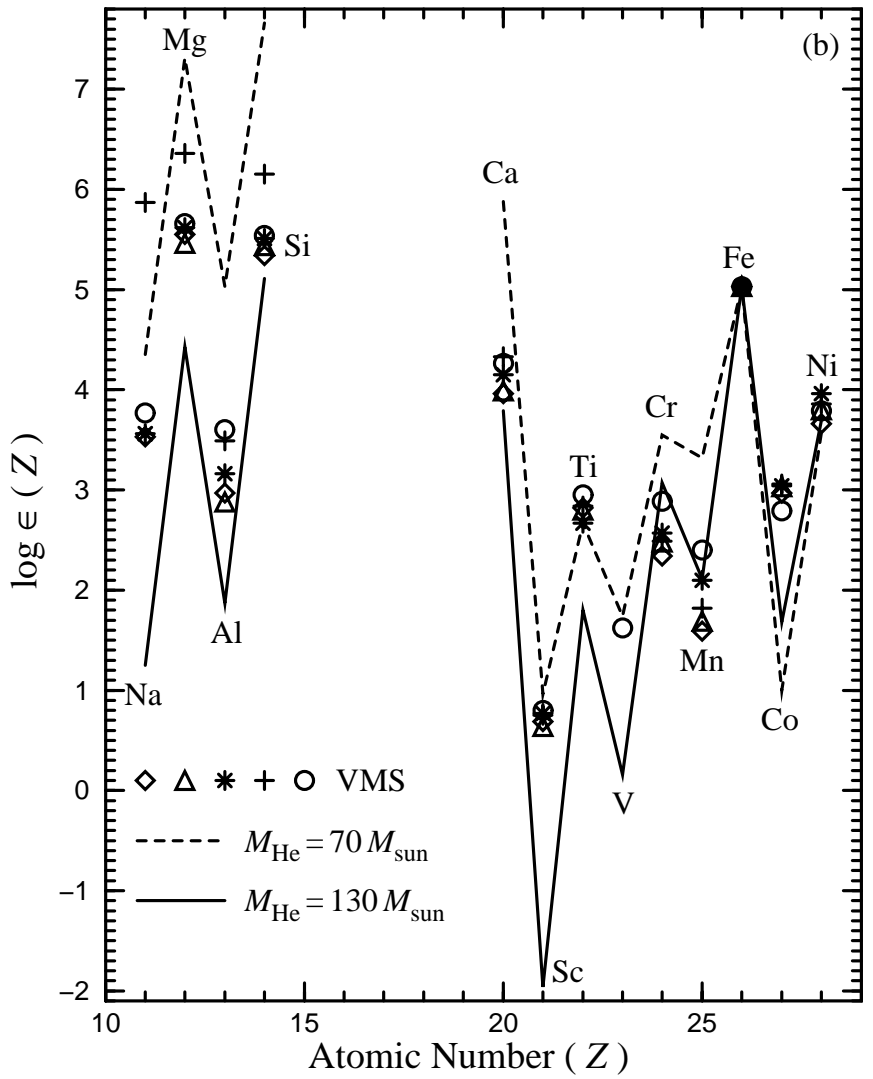

FIG. 9.-VMS yield patterns derived from the data on four stars with $[\mathrm{Fe} / \mathrm{H}]<-3$ and that derived from the $P$ inventory at $[\mathrm{Fe} / \mathrm{H}]=-3$ (same symbols as in Fig. 4) compared with the yield patterns calculated from models (Heger \& Woosley 2002) of VMSs with He core masses of $M_{\mathrm{He}}=70$ (dashed curve $)$ and $130 M_{\odot}($ solid curve $)$. The model yields have been shifted to pass through log $\epsilon(\mathrm{Mg})=5.58$ in panel $(a)$ and $\log \epsilon(\mathrm{Fe})=5.03$ in panel $(b)$. 
increases with the VMS mass. These values are larger than the typical SN II explosion energy of $\approx 10^{51}$ ergs by factors of $\sim 10-100$. From detailed numerical studies, Thornton et al. (1998) found that the total mass swept up by an SN remnant scales with the explosion energy to a power of 6/7. This suggests a dilution mass for VMSs that is $\sim 7-50$ times the standard dilution mass of $M_{\mathrm{dil}}^{\mathrm{SNII}} \approx 3 \times 10^{4} M_{\odot}$ for $\mathrm{SNe}$ II and is consistent with the lower end of the enormous dilution masses of $M_{\mathrm{dil}}^{\mathrm{VMS}} \sim 10^{6}-10^{7} M_{\odot}$ derived here for VMSs. Assuming that a single VMS gives $[\mathrm{Fe} / \mathrm{H}] \sim-4$ in a dilution mass of $\sim 10^{6} M_{\odot}$, we estimate that the Fe yield of VMSs is $\sim 0.1 M_{\odot}$. This also implies that $\sim 10$ VMSs had occurred in this dilution mass prior to the cessation of VMS activities at $[\mathrm{Fe} / \mathrm{H}] \approx-3$ (Qian \& Wasserburg 2001a concluded that many VMSs are required to provide the $P$ inventory of $\mathrm{O}$ ).

\subsubsection{Summary}

From the detailed ab initio nucleosynthetic models of SNe II and VMSs we would expect there to be a wide range in the relative production of the elements from $\mathrm{Na}$ to $\mathrm{Ni}$. However, we have shown in $\S 4$ that the evolution of the other elements relative to $\mathrm{Fe}$ over a wide range in $[\mathrm{Fe} / \mathrm{H}]$ can be adequately described by a phenomenological model with fixed yield patterns of SNe II $(L)$ and VMSs (see Fig. 6). This poses a serious dilemma. One obvious solution would be that the sampling of the ISM by each star represents contributions from many SNe II and VMSs whose average is strongly convergent. We well recognize that mixing can hide grossly diverse sources. However, it is not likely that the regular behavior exhibited by the data over the wide range in $[\mathrm{Fe} / \mathrm{H}]$ can be all attributed to mixing. For example, in the domain of $-2.5 \lesssim[\mathrm{Fe} / \mathrm{H}] \lesssim-2$, the abundances of the elements from $\mathrm{Na}$ to $\mathrm{Ni}$ should be dominated by the contributions from only several $\mathrm{SNe} \mathrm{II}(L)$. Were there diverse yield patterns among $\mathrm{SNe} \mathrm{II}(L)$, a convergent abundance pattern would not be obtained by averaging over so few events. However, the data in Figure 6 show that the relative abundances of $\mathrm{Mn}$ and $\mathrm{Co}$ to $\mathrm{Fe}$ already are well defined at $-2.5 \lesssim[\mathrm{Fe} / \mathrm{H}] \lesssim-2$. The data on other elements show the same behavior as can be found in the figures of McWilliam et al. (1995) and Norris et al. (2001).

While a statistical equilibrium among the $\alpha$-nuclei from ${ }^{28} \mathrm{Si}$ to, e.g., ${ }^{52} \mathrm{Fe}$ can be possibly at work to set a fixed yield pattern of the corresponding elements, there is no explanation, to our knowledge, of the yield patterns outlined here for $\mathrm{SNe} \mathrm{II}(L)$ and VMSs. The observed constancy of $\mathrm{Si} / \mathrm{Mg} \approx 1$ over a wide range in $[\mathrm{Fe} / \mathrm{H}]$ is in sharp contrast to what is found in models of SNe II and VMSs. We are pressed to inquire whether or not a new paradigm for SN II and VMS models should be explored that would treat the possibility of much more restrictive evolutionary paths for $\mathrm{SNe}$ II and VMSs. From the arguments presented here, the major efforts to model the solar abundance pattern by many sources with widely varying yield patterns have not led to a satisfactory conclusion. We recognize that ab initio calculations are both complex and difficult. It is possible that some unspecified dynamical effects involving partially quenched evolution through high-temperature and highdensity stages may be of importance in the production of $\mathrm{Mg}$ and Si. The phenomenological approach used here can in no way describe the detailed nuclear astrophysical evolution of SNe II or VMSs. However, we consider that the rather robust yield patterns of $\mathrm{SNe} \mathrm{II}(L)$ and VMSs are quite directly obtained from the observational data, and we cannot identify any obvious errors that would change the results. The only substantial variation is found for the VMS yields of $\mathrm{Na}, \mathrm{Mg}$, and $\mathrm{Si}$ from the data on one star (CS 22949-037). This lack of variation in SN II $(L)$ and VMS yields is remarkable in view of the different data sources used. We do not consider that this is plausibly attributable to some grand averaging. Instead, there may be possible mechanisms that would give sharply convergent yield patterns nearly independent of, e.g., the mass or metallicity of SN II progenitors. Certainly the observational data should serve as a guide to theoretical exploration of such mechanisms.

\subsection{Implications for Abundances at Ultralow Metallicities}

Based on the model presented here, the abundances at ultralow metallicities $([\mathrm{Fe} / \mathrm{H}]<-3)$ can be described in terms of the yield pattern of VMSs and the yields of $\mathrm{SNe}$ $\mathrm{II}(H)$ and $\mathrm{II}(L)$. For the elements from $\mathrm{Na}$ to $\mathrm{Ni}$, the dominant contributions are from VMSs. Thus, to first approximation, the observed abundances at $[\mathrm{Fe} / \mathrm{H}]<-3$ should follow the VMS yield pattern. A more detailed study can be carried out by using the $\mathrm{Ba}$ abundance as the index for $r$-process production to subtract the concomitant contributions from $\mathrm{SNe} \mathrm{II}(H)$ and $\mathrm{II}(L)$. Such a study may reveal more clearly the features at the elements of odd atomic numbers and $\mathrm{Cr}$ as these elements receive more contributions from $\mathrm{SNe} \mathrm{II}(L)$. All the $r$-process elements above $\mathrm{Sr}$ should be attributed to $\mathrm{SNe} \operatorname{II}(H)$ and $\mathrm{II}(L)$. The abundances of these elements at $[\mathrm{Fe} / \mathrm{H}]<-3$ may be complicated by the effects of discrete events and issues of the dilution mass. For example, a low-mass star might form in a region where an SN II $(H)$ just occurred but no nearby VMS explosion occurred yet. In this case, the star would sample the enrichments by an $\mathrm{SN} \mathrm{II}(H)$ with a standard dilution mass and thus show significant enhancements in the heavy $r$-process elements while maintaining an ultralow $[\mathrm{Fe} / \mathrm{H}]$. Note that a star sampling the enrichments by an SN II $(L)$ with a standard dilution mass would move out of the domain of ultralow metallicities as it would have $[\mathrm{Fe} / \mathrm{H}] \approx-2.5$ (see Fig. $5 b$ ). As $\mathrm{SNe} \mathrm{II}(H)$ are frequent, future observations at $[\mathrm{Fe} / \mathrm{H}]<-3$ may find stars with unusually high abundances of heavy $r$-process elements. In any case, given sufficient sensitivity, future observations may detect the heavy $r$-process elements such as Eu that were produced by $\mathrm{SNe} \mathrm{II}(H)$ concurrent with VMS activities at ultralow metallicities. It is evident that there is an urgent need for more extensive and precise observations of abundances in stars with metallicities well below -3 .

\subsection{Implications for Formation of Galaxies and Chemical Evolution of the Universe}

In considering broader implications of the results presented here, it should be noted that the conclusions are only based on observational data and a phenomenological model. No direct considerations are made of cosmological models or the dynamics and mechanisms associated with SN and VMS evolution. Time is, for the most part, only considered in terms of sequence. Increments in the inventory of elements associated with newer generations of stars are taken as the measure of time sequence. The other guiding rules are the general aspects of stellar evolution.

The following general evolution sequence is inferred: (1) formation of VMSs made of big bang debris, (2) concomi- 
tant formation at some level of a normal stellar population, (3) "metal" enrichment with overwhelming contributions from VMSs until a metallicity of $[\mathrm{Fe} / \mathrm{H}] \approx-3$ was reached, (4) cessation of VMS activities and onset of major formation of normal stellar populations as marked by a sharp increase of contributions from the frequent $\mathrm{SNe} \mathrm{II}(H)$ at $[\mathrm{Fe} / \mathrm{H}] \approx-3,(5)$ continued chemical enrichment with dominant contributions from $\mathrm{SNe} \mathrm{II}(H)$ and $\mathrm{II}(L)$ until $[\mathrm{Fe} / \mathrm{H}] \sim-1$ was reached, (6) onset of SN Ia contributions at $[\mathrm{Fe} / \mathrm{H}] \sim-1$ when a sufficient number of low-mass stars had evolved in binaries to serve as precursors for $\mathrm{SNe}$ Ia, and (7) continued chemical enrichment with contributions from $\mathrm{SNe} \mathrm{II}(H), \mathrm{II}(L)$, Ia and other evolved low-mass stars. In the regime of $[\mathrm{Fe} / \mathrm{H}]<-3$ where VMSs play a predominant role, we infer that in aggregations of matter where astration has begun, the explosion of a VMS redistributes and mixes matter over a mass scale of $\sim 10^{6}-10^{7}$ $M_{\odot}$. This involves fresh nucleosynthetic products of the VMS and any SN II debris in the region. Thus, when VMS activities cease at $[\mathrm{Fe} / \mathrm{H}] \approx-3$, the dilution mass with which nucleosynthetic products are mixed drops by a few orders of magnitude from $\sim 10^{6}-10^{7} M_{\odot}$ for $[\mathrm{Fe} / \mathrm{H}]<-3$ to the value of $\approx 3 \times 10^{4} M_{\odot}$ associated with SNe II for $[\mathrm{Fe} / \mathrm{H}] \gtrsim-3$.

In typical cosmological models of hierarchical structure formation, the condensation of dark matter is considered to provide the gravitational potential wells in which some baryonic matter collects. The typical binding energies of these wells correspond to escape velocities of $\sim 100 \mathrm{~km} \mathrm{~s}^{-1}$. The VMS explosions are more than sufficient to disrupt the baryonic condensates in such wells. We consider that in the regime of $[\mathrm{Fe} / \mathrm{H}]<-3$, the increases in "metallicity" are due to the sequence of local condensation of baryonic matter, formation of VMSs and some normal stars, and dispersion by VMS explosion. There are then later reaggregations of some baryonic matter and further enrichments through repeating the above sequence. The cessation of VMS activities is due to the increase in metallicity that favors formation of stars with a more normal distribution in mass. This permits the aggregation of some matter without disruption. Subsequent to the cessation of VMS activities at $[\mathrm{Fe} / \mathrm{H}] \approx-3$, large-scale dispersion of baryonic matter becomes very limited and large aggregation of baryonic matter that leads to normal galactic structures and galactic chemical evolution begins to occur. A recent study by Bromm et al. (2001a) indicates that substantial formation of a normal stellar population could only occur when the metallicity is substantially above $5 \times 10^{-4}$ times the solar value.

In considering the domain where VMSs and $\mathrm{SNe}$ II coexist (region A in Fig. 5), it is necessary to discuss the timing of events. Based on the difficulty of forming stars with lower masses from big bang debris (e.g., Bromm et al. 2001a), the first objects formed would have to be VMSs. Upon explosion these would disrupt the original baryonic aggregates formed in the potential wells of dark matter. Reaggregation and repetition of such events would increase the metallicity to allow formation of both VMSs and lower mass stars. Certainly some lower mass stars began to form by $[\mathrm{Fe} / \mathrm{H}] \sim-4$. Consider a "cloud" which contains VMSs and SN II progenitors. The VMSs would explode first and disrupt the cloud. The subsequent SN II explosions would disperse ejecta into a hot and tenuous medium that does not permit any immediate star formation. After suffi- cient cooling, the ejecta from the VMSs and SNe II and the general medium would reaggregate under the influence of the dark matter potential. During this process, the nucleosynthetic products of VMSs and SNe II would be effectively mixed over the entire mass of the baryonic aggregate prior to astration. Thus, the effective dilution mass for SNe II (much larger than the standard dilution mass) is the same as that for VMSs in the regime where VMSs are active. When $[\mathrm{Fe} / \mathrm{H}] \approx-3$ is reached, astration into lower mass stars becomes very efficient and formation of VMSs is truncated. The total timescale over which normal astration becomes dominant is not known. Based on considerations of data on damped Ly $\alpha$ systems, Wasserburg \& Qian (2000b) estimated that this timescale was typically several times $10^{9} \mathrm{yr}$ after the big bang. The question of reaggregation is a complex one as it involves condensation from ionized matter. It is known that most of the baryonic matter resides in the ionized intergalactic medium (IGM). This matter has not yet formed and may never form galaxies.

Observations of damped $L y \alpha$ systems over a wide range in redshift $(z \approx 1.5-4.5)$ show that the lowest $[\mathrm{Fe} / \mathrm{H}]$ observed is approximately -2.7 (e.g., Prochaska \& Wolfe 2000; Prochaska, Gawiser, \& Wolfe 2001). The damped Ly $\alpha$ systems are thought to represent protogalaxies. The lower bound on $[\mathrm{Fe} / \mathrm{H}]$ in these systems was interpreted to reflect the transition to normal astration at $[\mathrm{Fe} / \mathrm{H}] \approx-3$ over an extended timescale after the big bang (Wasserburg \& Qian 2000b). As this is the same effective bound for a wide range of $z$, it follows that subsequent to the cessation of VMS activities at $[\mathrm{Fe} / \mathrm{H}] \approx-3$, most of the baryonic matter remained dispersed in the universe to serve as a reservoir for formation of protogalaxies. Based on the model presented here, this matter should exhibit the chemical enrichments corresponding to the $P$ inventory that resulted from the integrated production by VMSs and some $\mathrm{SNe}$ II prior to the achievement of $[\mathrm{Fe} / \mathrm{H}] \approx-3$. Assuming the yields of Heger \& Woosley (2002), Oh et al. (2001) have shown that a fraction $\sim 10^{-5}$ to $10^{-4}$ of all baryonic matter must be processed through VMSs in order to account for the $P$ inventory at $[\mathrm{Fe} / \mathrm{H}] \approx-3$. Using the VMS luminosities of Bromm, Kudritzki, \& Loeb (2001b), they have further argued that this amount of processing would provide $\sim 10$ photons per baryon at energies sufficient to ionize $\mathrm{H}$ and He. This suggests that VMSs may be sufficient to explain the Gunn-Peterson effect (Gunn \& Peterson 1965), which requires that most of the baryonic matter be ionized. How effective the ionization by VMSs may be for $[\mathrm{Fe} / \mathrm{H}]$ substantially below -3 is not yet explored. The $P$ inventory given here is thus considered by us to represent the abundances in dispersed ionized baryonic matter and should be compared with what is observed in, e.g., the IGM. If an acceleration mechanism were to exist in the dispersed ionized baryonic matter, a cosmic-ray component might be produced with a composition reflecting the $P$ inventory.

It has long been recognized that the ratio $\mathrm{E} / \mathrm{Fe}$ for the $\alpha$-elements such as $\mathrm{Mg}, \mathrm{Si}$, and $\mathrm{Ca}$ in the early Galaxy is higher than the solar value by a factor of $\approx 3$. This was due to the additional $\mathrm{Fe}$ production by $\mathrm{SNe}$ Ia at later times (e.g., Tinsley 1980). Only $\approx \frac{1}{3}$ of the solar Fe inventory was produced by SNe II. The ratio $\mathrm{E} / \mathrm{Fe}$ for the $\alpha$-elements in the early Galaxy was considered to reflect the production of these elements by SNe II. We have shown that the production of these elements at $[\mathrm{Fe} / \mathrm{H}]<-3$ was dominated by VMSs. However, the yield pattern of $\mathrm{Mg}, \mathrm{Si}, \mathrm{Ca}, \mathrm{Ti}$, and 
$\mathrm{Fe}$ for VMSs is almost identical to that for $\mathrm{SNe} \mathrm{II}(L)$. Thus, the ratio $\mathrm{E} / \mathrm{Fe}$ for the $\alpha$-elements stays approximately constant prior to the onset of SN Ia contributions although the dominant production sources have changed at $[\mathrm{Fe} / \mathrm{H}] \approx$ -3 as a result of the cessation of VMS activities. The special status of $[\mathrm{Fe} / \mathrm{H}] \approx-3$ is demonstrated by the sharp increase in $\mathrm{Ba}$ abundance at this metallicity (the same sharp increase also occurs at, e.g., $[\mathrm{Si} / \mathrm{H}] \approx-2.5$ as $[\mathrm{Si} / \mathrm{Fe}] \approx 0.5$ at low $[\mathrm{Fe} / \mathrm{H}]$; see Fig. 2 of $\mathrm{Oh}$ et al. 2001). The cosmological epoch prior to the achievement of $[\mathrm{Fe} / \mathrm{H}] \approx-3$ remains to be explored.
We would like to thank Stan Woosley for education on nucleosynthesis in SNe and VMSs and Volker Bromm for his penetrating questions. We also would like to thank an anonymous referee for helpful comments. Y.-Z. Q. acknowledges the hospitality of T-16 at the Los Alamos National Laboratory during the initial phase of this work. This work was supported in part by DOE grants DE-FG0287ER40328 and DE-FG02-00ER41149 (Y. -Z. Q.) and by NASA grant NAG 5-10293 (G. J. W.), Caltech Division Contribution 8770(1081).
Anders, E., \& Grevesse, N. 1989, Geochim. Cosmochim. Acta, 53, 197

Arlandini, C., Käppler, F., Wisshak, K., Gallino, R., Lugaro, M., Busso, M., \& Straniero, O. 1999, ApJ, 525, 886

Benetti, S., et al. 2001, MNRAS, 322, 361

Bond, J. R., Arnett, W. D., \& Carr, B. J. 1984, ApJ, 280, 825

Bromm, V., Ferrara, A., Coppi, P. S., \& Larson, R. B. 2001a, MNRAS, submitted

Bromm, V., Kudritzki, R. P., \& Loeb, A. 2001b, ApJ, 552, 464

Burris, D. L., Pilachowski, C. A., Armandroff, T. E., \& Sneden, C. 2000, ApJ, 544, 302

Cayrel, R., et al. 2001, Nature, 409, 691

Glatzel, W., Fricke, K. J., \& El Eid, M. F. 1985, A\&A, 149, 413

Gratton, R. G. 1989, A\&A, 208, 171

Gratton, R. G., \& Sneden, C. 1988, A\&A, 204, 193 . 1991, A\&A, 241, 501

Gunn, J. E., \& Peterson, B. A. 1965, ApJ, 142, 1633

Heger, A., \& Woosley, S. E. 2002, ApJ, in press

Hillebrandt, W., Nomoto, K., \& Wolff, R. G. 1984, A\&A, 133, 175

Hoffman, R. D., Woosley, S. E., \& Qian, Y.-Z. 1997, ApJ, 482, 951

Mayle, R. W., \& Wilson, J. R. 1988, ApJ, 334, 909

McWilliam, A. 1998, AJ, 115, 1640

McWilliam, A., Preston, G. W., Sneden, C., \& Searle, L. 1995, AJ, 109, 2757

Nakamura, T., Umeda, H., Nomoto, K., Thielemann, F.-K., \& Burrows, A. 1999, ApJ, 517, 193

Nomoto, K. 1984, ApJ, 277, 791

Norris, J. E., Ryan, S. G., \& Beers, T. C. 2001, ApJ, 561, 1034

\section{REFERENCES}

Oh, S. P., Nollett, K. M., Madau, P., \& Wasserburg, G. J. 2001, ApJ, 561, L1

Prochaska, J. X., Gawiser, E., \& Wolfe, A. M. 2001, ApJ, 552, 99

Prochaska, J. X., \& Wolfe, A. M. 2000, ApJ, 533, L5

Qian, Y.-Z., \& Wasserburg, G. J. 2001a, ApJ, 549, 337 $2001 \mathrm{~b}$, ApJ, 552, L55 2001c, ApJ, 559, 925 (QW01c)

Qian, Y.-Z., \& Woosley, S. E. 1996, ApJ, 471, 331

Rakavy, G., Shaviv, G., \& Zinamon, Z. 1967, ApJ, 150, 131

Ryan, S. G., Norris, J. E., \& Beers, T. C. 1996, ApJ, 471, 254

Sneden, C., Cowan, J. J., Ivans, I. I., Fuller, G. M., Burles, S., Beers, T. C., \& Lawler, J. E. 2000, ApJ, 533, L139

Thielemann, F.-K., Nomoto, K., \& Hashimoto, M. 1996, ApJ, 460, 408

Thornton, K., Gaudlitz, M., Janka, H.-Th., \& Steinmetz, M. 1998, ApJ, 500,95

Timmes, F. X., Woosley, S. E., \& Weaver, T. A. 1995, ApJS, 98, 617

Tinsley, B. M. 1980, Fundam. Cosmic Phys., 5, 287

Wasserburg, G. J., Busso, M., \& Gallino, R. 1996, ApJ, 466, L109

Wasserburg, G. J., \& Qian, Y.-Z. 2000a, ApJ, 529, L21 (WQ00a)

.2000b, ApJ, 538, L99

Westin, J., Sneden, C., Gustafsson, B., \& Cowan, J. J. 2000, ApJ, 530, 783

Wheeler, J. C., Cowan, J. J., \& Hillebrandt, W. 1998, ApJ, 493, L101

Woosley, S. E., \& Weaver, T. A. 1995, ApJS, 101, 181

Woosley, S. E., Wilson, J. R., Mathews, G. J., Hoffman, R. D., \& Meyer, B. S. 1994, ApJ, 433, 229 